Document downloaded from:

http://hdl.handle.net/10251/105486

This paper must be cited as:

Burgos, C.; Cortés, J.; Villafuerte, L.; Villanueva Micó, RJ. (2017). Extending the deterministic Riemann-Liouville and Caputo operators to the random framework: A mean square approach with applications to solve random fractional differential equations. Chaos, Solitons and Fractals. 102:305-318. doi:10.1016/j.chaos.2017.02.008

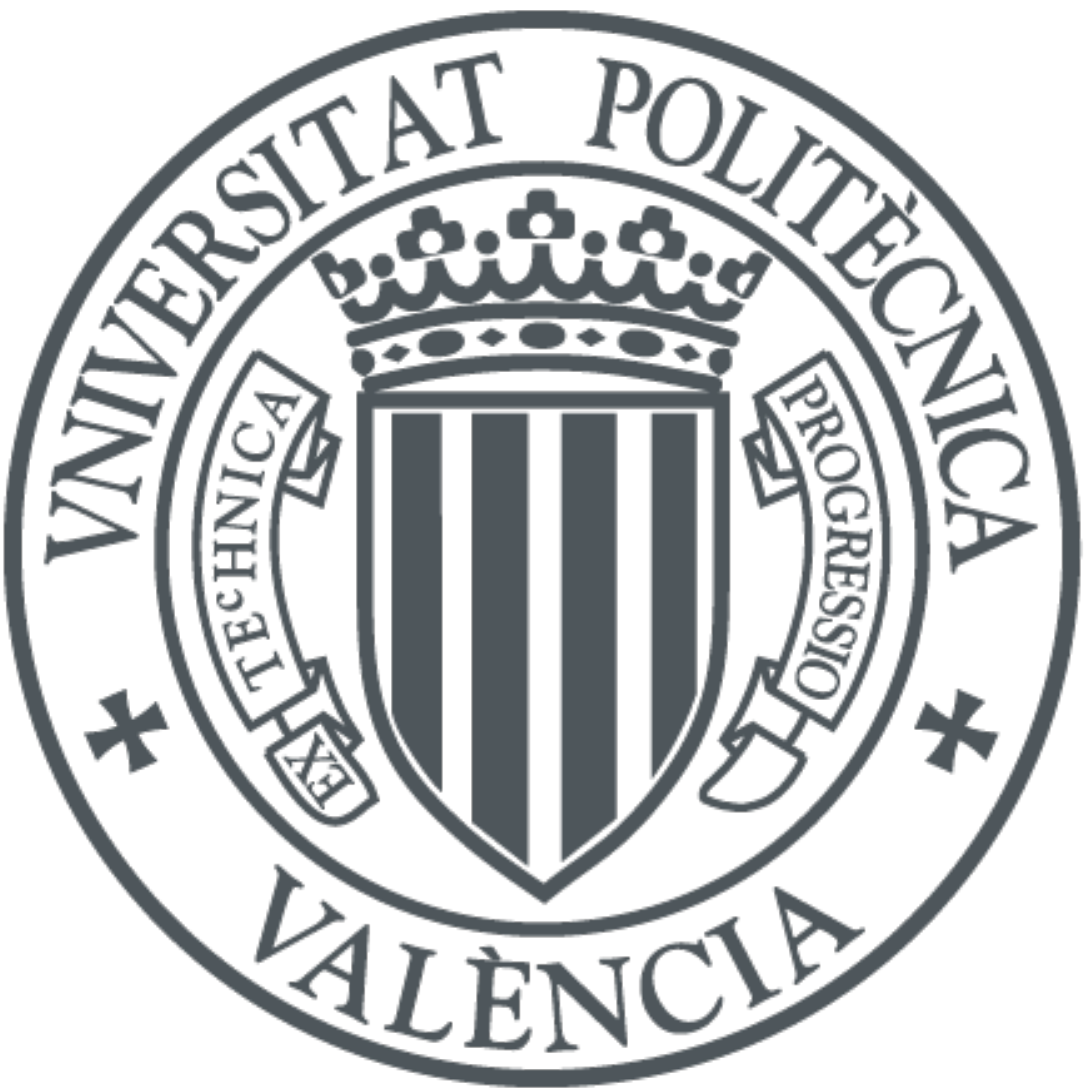

The final publication is available at

https://doi.org/10.1016/j.chaos.2017.02.008

Copyright Elsevier

Additional Information 


\title{
Extending the deterministic Riemann-Liouville and Caputo operators to the random framework: A mean square approach with applications to solve random fractional differential equations
}

\author{
C. Burgos ${ }^{\mathrm{a}}$, J.-C. Cortés ${ }^{\mathrm{a}}$, L. Villafuerte ${ }^{\mathrm{b}}$, R.-J. Villanueva ${ }^{\mathrm{a}}$ \\ ${ }^{a}$ Instituto Universitario de Matemática Multidisciplinar, \\ Universitat Politècnica de València, \\ Camino de Vera s/n, 46022, Valencia, Spain \\ ${ }^{b}$ Facultad de Ciencias en Físicas y Matemáticas, \\ Universidad Autónoma de Chiapas, Tuxtla Gutiérrez, Chiapas,Mexico \\ Department of Mathematics, \\ University of Texas at Austin, USA
}

\begin{abstract}
This paper extends both the deterministic fractional Riemann-Liouville integral and the Caputo fractional derivative to the random framework using the mean square random calculus. Characterizations and sufficient conditions to guarantee the existence of both fractional random operators are given. Assuming mild conditions on the random input parameters (initial condition, forcing term and diffusion coefficient), the solution of the general random fractional linear differential equation, whose fractional order of the derivative is $\alpha \in] 0,1]$, is constructed. The approach is based on a mean square chain rule, recently established, together with the random Fröbenius method. Closed formulae to construct reliable approximations for the mean and the covariance of the solution stochastic process are also given. Several examples illustrating the theoretical results are included.
\end{abstract}

Keywords: Random mean square Riemann-Liouville integral, random mean square Caputo derivative, random fractional linear differential equation, random Fröbenius method.

\section{Introduction}

The goal of this paper is twofold. Firstly, to extend some important concepts and results that belong to the deterministic fractional calculus to the random framework using the so-called mean square approach. Secondly, to show some applications of the mean square random fractional calculus to solve fractional differential equations with uncertainties. To start with, we will give a motivation of our study in connection with the different available approaches to deal with differential equations with randomness.

Nowadays, it is widely accepted that the behaviour of many physical phenomena is governed by chance. Thus, it is not appropriate to describe them just using deterministic physical laws

${ }^{*}$ Corresponding author

Email addresses: clabursi@posgrado.upv.es (C. Burgos), jccortes@imm.upv.es (J.-C. Cortés), lva5@hotmail.com (L. Villafuerte), rjvillan@imm.upv.es (R.-J. Villanueva) 
but considering the randomness into the physical formulations. In this regard, it is well-known that the trajectory of a rocket is determined by the randomness of the initial speed; the electric power exhibits visible irregular changes that behave irregularly; the value of assets in financial markets is often very volatile, just as a few examples where it is reasonable to consider uncertainty. From this point of view, it is natural to take advantage of the powerful effectiveness of deterministic differential equations for describing physical phenomena and consider uncertainty in their formulation. This leads to two different approaches, namely, stochastic differential equations (SDEs) and random differential equations (RDEs). While there is still in the scientific community a wrong tendency to treat these two terms as synonymous, it is important to point out that they are distinctly different and they require completely different techniques for analysis and treatment [1]. The main difference between SDEs and RDEs comes from the kind of the uncertainty that is considered in the formulation of both equations. On the one hand, SDEs are forced by an irregular stochastic process such as a Wiener process (also termed brownian motion). This is a gaussian stochastic process whose sampled trajectories are nowhere differentiable. Solving SDEs requires of a special stochastic calculus, usually referred to as Itô Calculus, whose cornerstone is the Itô lemma [2]. On the other hand, RDEs are those in which the random effects are manifested directly via the input parameters (coefficients, source terms and initial and boundary conditions). Under this approach it is assumed that input parameters possess milder or regular sample behaviour (e.g., sample continuity or sample differentiability, etc). Apart from gaussian distribution, many other important probability distributions are allowed to have the input parameters (binomial, Poisson, beta, gamma, etc). This latter feature makes RDEs very attractive when modelling physical phenomena since they permit the consideration of uncertainty in their formulation. Both SDEs and RDEs have demonstrated to be powerful tools in dealing with important theoretical and practical mathematical problems (see [3, 4] for SDEs, and [5, 6, 7, 8] for RDEs, for instance).

Throughout this paper will be considered RDEs only. Some recent contributions about RDEs are $[9,10,11,12]$. It is important to point out that there are different approaches to deal with RDEs, but in these pages we will follow the so-called mean square approach [8]. This approach is based upon a strong stochastic type-convergence, termed mean square convergence, whose main advantage is that the results established in mean square are also valid in other important types of stochastic convergences, namely, convergence in probability and convergence in distribution. Additionally, the mean square convergence possesses a distinctive property, which will be used in this paper (see Proposition 10), that makes it especially suitable to construct reliable approximations of the mean and variance of the solution stochastic process of RDEs. Some recent papers where RDEs are studied using the mean square calculus are $[13,14,15,16]$, for instance.

Over the last few decades deterministic fractional differential equations are having an important impact on both the theory and applications of mathematics. Despite their physical meaning of the fractional derivative is not still clear, fractional differential equations are gaining influence in mathematical modelling because their success in modelling phenomena having a microscopic complex behaviour whose macroscopic dynamics can not be properly described using the classical deterministic derivative. Some areas where deterministic fractional differential equations have demonstrated to be useful tools include Viscoelasticity Materials, Fluid Flows, Solute Transport, etc., [17]. Many author attribute the success of fractional differential equations to the fact that many of the physical processes related to complex systems possess non-local dynamics involving long-memory in time, and the fractional integral and fractional derivative operators do have some of those characteristics $[17,18,19]$. So, it is natural to introduce randomness into the 
mathematical formulation of fractional differential equations. While a number of contributions have dealt with fractional SDEs [20, 21], for example, to the best of our knowledge there is a lack of study regarding fractional RDEs. Some noteworthy exceptions have been recently presented in $[22,23]$. In these two contributions interesting existence and uniqueness results, based on the so-called sample path and $\mathrm{L}^{p}$-approaches, for initial value problems formulated through fractional RDEs have been presented.

Finally, it is important to point out that there is a number of fractional derivatives such as Caputo, Riemann-Liouville, Grünwald-Letnikov [24, 25]. In this paper we will only consider the Caputo derivative since we are interested in constructing a mean square solution to the general fractional linear first-order differential equation with random coefficients and random initial condition. The Caputo fractional derivative has the key property of allowing to express initial conditions in terms of the classical derivatives.

This paper is organized as follows. Section 2 contains the main results related to the socalled $\mathrm{L}^{2}$-random calculus, also termed mean square calculus that will be required throughout this paper. In Section 3, we extend the concept of the fractional Riemann-Liouville integral and fractional Caputo derivative to the mean square random calculus. Characterizations of these two important random fractional operators, in terms of the correlation function of the involved second-order stochastic process, are explicitly given. Section 4 is addressed to show how the random Fröbenius power series method can be applied to successfully solve the complete random linear fractional differential equation under very general hypotheses and assuming randomness in all its input parameters (initial condition, forcing term and diffusion coefficient). General explicit formulaes for computing accurate approximations of the mean, variance and covariance functions of the solution stochastic process to the complete random linear fractional differential equation are given in Section 5. Section 6 is devoted to exhibit several illustrative examples. Conclusions are drawn in Section 7.

\section{Preliminaries about mean square random calculus}

For the sake of completeness, henceforth we will summarize the main definitions and results that will be used throughout this paper. A comprehensive survey of them can be found in [2, ch.1], [26, ch.XI], [8, ch.4] and [27]. A complex random variable (RV), $X: \Omega \rightarrow \mathbb{C}$, defined on a complete probability space $(\Omega, \mathfrak{F}, \mathbb{P})$ is said to be of order $p \geq 1$ ( $p$-RV, for short), if $\mathbb{E}\left[|X|^{p}\right]<+\infty$, where $\mathbb{E}[\cdot]$ denotes the expectation operator. The space $\mathrm{L}^{p}(\Omega)$ of all $p$-RVs endowed with the norm

$$
\|X\|_{p}=\left(\mathbb{E}\left[|X|^{p}\right]\right)^{1 / p},
$$

is a Banach space [2, p.9]. The convergence in $\mathrm{L}^{p}(\Omega)$, usually called convergence in $p$-th mean, it is naturally inferred by the $p$-norm (1), i.e., a sequence of $\operatorname{RVs}\left\{X_{n}: n \geq 0\right\}$ in $\mathrm{L}^{p}(\Omega)$ is said to be $p$-th mean convergent to $X \in \mathrm{L}^{p}(\Omega)$ if, and only if, $\left\|X_{n}-X\right\|_{p} \underset{n \rightarrow+\infty}{\longrightarrow} 0$. Given $\emptyset \neq \mathcal{U} \subset \mathbb{R}$, a family of RVs indexed by $u \in \mathcal{U}, X(u) \equiv\{X(u): u \in \mathcal{U}\}$ is called a stochastic process (SP). Throughout this paper, we will take $\mathcal{U}=\left[0,+\infty\left[\right.\right.$. If $\mathbb{E}\left[|X(u)|^{p}\right]<+\infty$ for each $u \in \mathcal{U}$, then $X(u)$ is said to be a $p$-stochastic process ( $p$-SP, for short). The definitions of $p$-continuity, $p$ differentiability and $p$-integrability of $p$-SPs in $\mathrm{L}^{p}(\Omega)$-spaces are the usual ones derived from the $p$-norm (1) in Banach spaces. A significant case corresponds to $p=2$. In fact, it can be seen that $\left(\mathrm{L}^{2}(\Omega),\|\cdot\|_{2}\right)$ is a Hilbert space with the inner product

$$
\langle X, Y\rangle=\mathbb{E}[|X Y|], \quad X, Y \in \mathrm{L}^{2}(\Omega),
$$


being

$$
\|X\|_{2}=+\sqrt{\mathbb{E}\left[|X|^{2}\right]} .
$$

The convergence associated to this $\|\cdot\|_{2}$-norm is usually referred to as mean square convergence. Hereinafter, $X_{n} \underset{n \rightarrow+\infty}{\stackrel{\text { m.s. }}{\longrightarrow}} X$ will denote a sequence, $\left\{X_{n}: n \geq 0\right\}$, of $\mathrm{RVs}_{\text {in }} \mathrm{L}^{2}(\Omega)$ such that is mean square convergent to $X$ as $n \rightarrow+\infty$. As it shall see later, the Schwarz's inequality

$$
\mathbb{E}[|X Y|] \leq\|X\|_{2}\|Y\|_{2}, \quad X, Y \in \mathrm{L}^{2}(\Omega),
$$

will be used extensively throughout the paper. Another important inequality that will be subsequently applied is the Jensen's inequality. If $f: \mathbb{R} \longrightarrow \mathbb{R}$ is a convex mapping and $X$ is a $\mathrm{RV}$, then

$$
f(\mathbb{E}[X]) \leq \mathbb{E}[f(X)],
$$

provided all the above involved moments exist.

Although mean square convergence is an important type of stochastic convergence, some basic operational rules do not fulfil unless additional hypotheses are assumed. Now, we prove a result in this respect that will be required later.

Proposition 1. Let $X$ be a bounded $R V$ in $\mathrm{L}^{2}(\Omega)$, i.e., there exist constants $x_{1}$ and $x_{2}$ such that $x_{1} \leq X(\omega) \leq x_{2}$, for all $\omega \in \Omega$, and let us assume that $Z_{n}$ converges in the mean square sense to Z. Then, $X Z_{n} \underset{n \rightarrow \infty}{\stackrel{m . s .}{\longrightarrow}} X Z$.

Proof. Let $\hat{x}=\max \left\{\left|x_{1}\right|,\left|x_{2}\right|\right\}<+\infty$, and observe that

$$
0 \leq\left(\left\|X Z_{n}-X Z\right\|_{2}\right)^{2}=\mathbb{E}\left[|X|^{2}\left|Z_{n}-Z\right|^{2}\right] \leq|\hat{x}|^{2} \mathbb{E}\left[\left|Z_{n}-Z\right|^{2}\right]=|\hat{x}|^{2}\left(\left\|Z_{n}-Z\right\|_{2}\right)^{2} \underset{n \rightarrow+\infty}{\longrightarrow} 0,
$$

since $\left\{Z_{n}\right\}$ converges in the mean square sense to $Z$ as $n \rightarrow+\infty$. Then, the result is proved.

Another property that will be used throughout the paper is the following

Proposition 2. [28, p.92] Let $f, g: \mathbb{R} \longrightarrow \mathbb{R}$ be measurable mappings and $X, Y: \Omega \longrightarrow \mathbb{R}$ independent $R V$ s. Then, $f(X)$ and $g(Y)$ are independent $R V s$ and

$$
\mathbb{E}[f(X) g(Y)]=\mathbb{E}[f(X)] \mathbb{E}[g(Y)],
$$

provided the above expectations exist.

As we shall see below, the concept of mean square differentiable 2-SP will be required for introducing the random mean square fractional Caputo derivative. We recall that a 2-SP $\{X(u): u \in$ $\mathcal{U}\}$ has a mean square derivative $\frac{\mathrm{d} X(u)}{\mathrm{d} u}$ at $u \in \mathcal{U}$ if

$$
\lim _{\tau \rightarrow 0}\left\|\frac{X(u+\tau)-X(u)}{\tau}-\frac{\mathrm{d} X(u)}{\mathrm{d} u}\right\|_{2}=0, \quad u, u+\tau \in \mathcal{U} .
$$

Higher order mean square derivatives, denoted by $\frac{\mathrm{d}^{n} X(u)}{\mathrm{d} u^{n}} \equiv X^{(n)}(u), n \geq 1$, are defined analogously.

When two o more RVs are involved, statistical dependence is an important matter. To deal with statistical dependence it is convenient to introduce the definition of correlation function. If $X(u)$ is a 2-SP, then for each $u_{1}, u_{2}>0$, the two-dimensional deterministic function $\Gamma_{X}\left(u_{1}, u_{2}\right)=$ 
$117 \mathbb{E}\left[X\left(u_{1}\right) X\left(u_{2}\right)\right]$ is called the correlation function associated to $X(u)$. The correlation function ${ }_{118} \Gamma_{X}\left(u_{1}, u_{2}\right)$ of a 2-SP $X(u)$ always exists since

$$
\left|\Gamma_{X}\left(u_{1}, u_{2}\right)\right|=\left|\mathbb{E}\left[X\left(u_{1}\right) X\left(u_{2}\right)\right]\right| \leq \mathbb{E}\left[\left|X\left(u_{1}\right) X\left(u_{2}\right)\right|\right] \leq\left\|X\left(u_{1}\right)\right\|_{2}\left\|X\left(u_{2}\right)\right\|_{2}<+\infty .
$$

Notice that in the latter expression, we have applied first the Jensen inequality (4) with $f(x)=$ $|x|$, which is a convex function, and secondly, the Schwarz's inequality (3). Finally, since $X\left(u_{1}\right), X\left(u_{2}\right) \in \mathrm{L}^{2}(\Omega)$, hence the norms $\left\|X\left(u_{1}\right)\right\|_{2}$ and $\left\|X\left(u_{2}\right)\right\|_{2}$ are finite.

We point out that many mean square properties, such as $\|\cdot\|_{2}$-continuity, $\|\cdot\|_{2}$-differentiability and $\|\cdot\|_{2}$-integrability of a 2 -SP, say $X(u)$, can be directly characterized through its correlation function $\Gamma_{X}\left(u_{1}, u_{2}\right)$ [8, ch.4].

Apart from the correlation function, other important functions that will be used in the subsequent sections to study the statistical dependence of the involved RVs are the covariance function, $\mathbb{C}_{X}\left(u_{1}, u_{2}\right)$ of 2-S.P. $X(u)$, and the cross-covariance function, $\mathbb{C}_{X, Y}\left(u_{1}, u_{2}\right)$, of two second-order SPs $X(u)$ and $Y(u)$. These functions are defined by

$$
\begin{aligned}
\mathbb{C}_{X}\left(u_{1}, u_{2}\right) & =\Gamma_{X}\left(u_{1}, u_{2}\right)-\mathbb{E}\left[X\left(u_{1}\right)\right] \mathbb{E}\left[X\left(u_{2}\right)\right], \\
\mathbb{C}_{X, Y}\left(u_{1}, u_{2}\right) & =\mathbb{E}\left[X\left(u_{1}\right) Y\left(u_{2}\right)\right]-\mathbb{E}\left[X\left(u_{1}\right)\right] \mathbb{E}\left[Y\left(u_{2}\right)\right],
\end{aligned}
$$

respectively.

We have seen that the mean square derivative of a 2-SP, say $X(u)$, and its higher order ones, if they exist are also 2-SPs. It can be shown that their correlation functions are determined simply in terms of the correlation function of $X(u)$ [8, p.97]. Specifically, if $X(u)$ is $n$-times mean square differentiable, then

$$
\Gamma_{X^{(n)}}\left(u_{1}, u_{2}\right)=\frac{\partial^{2 n} \Gamma_{X}\left(u_{1}, u_{2}\right)}{\partial u_{1}^{n} \partial u_{2}^{n}} .
$$

The following result gives a characterization of the existence of the mean square Riemann integral of a 2-SP, in terms of the existence of a two-dimensional integral involving the correlation function of the 2-SP.

Proposition 3. ([8, Th. 4.5.1]) Let $g(u, w)$ be a deterministic Riemann integrable function on the real interval $u \in[c, d]$, for every $w \in W \subset \mathbb{R}$, and let $X(u)$ be a 2-SP. Then, the 2-SP defined by

$$
Y(w)=\int_{c}^{d} g(u, w) X(u) \mathrm{d} u, \quad w \in \mathcal{W},
$$

exists if and only if, the deterministic double Riemann integral

$$
\int_{c}^{d} \int_{c}^{d} g\left(u_{1}, w\right) g\left(u_{2}, w\right) \Gamma_{X}\left(u_{1}, u_{2}\right) \mathrm{d} u_{1} \mathrm{~d} u_{2},
$$

exists and is finite.

The following consequence of the previous proposition will be used later.

Remark 1. In the particular case that $w=d \in \mathcal{W} \subset \mathbb{R}$ in Proposition 3 , the RV

$$
Y \equiv Y(d)=\int_{c_{5}}^{d} g(u, d) X(u) \mathrm{d} u
$$


is well-defined as a 2-RV, if and only if, the deterministic double Riemann integral

$$
\int_{c}^{d} \int_{c}^{d} g\left(u_{1}, d\right) g\left(u_{2}, d\right) \Gamma_{X}\left(u_{1}, u_{2}\right) \mathrm{d} u_{1} \mathrm{~d} u_{2},
$$

exists and is finite.

A key result, that will be used in this paper to construct a random generalized power series solution to the random fractional linear differential equation with a random initial condition, is the following chain rule [29]. This rule allows us to compute the mean square derivative of a 2-SP resulting from the composition of a differentiable deterministic function and a mean square differentiable 2-SP.

Theorem 1. Let $g$ be a deterministic continuous function on $\left[a_{1}, a_{2}\right]$ such that $g^{\prime}(t)$ exists and is finite at some point $t \in\left[a_{1}, a_{2}\right]$. If $\{X(v): v \in \mathcal{V}\}$ is a 2-SP such that

i) The interval $\mathcal{V}$ contains the range of $g, g\left(\left[a_{1}, a_{2}\right]\right) \subset \mathcal{V}$.

ii) $X(v)$ is mean square differentiable at the point $g(t)$.

iii) The mean square derivative of $X(v), \frac{\mathrm{d} X(v)}{\mathrm{d} v}$, is mean square continuous on $\mathcal{V}$.

Then, the 2-SP, $X(g(t))$, is mean square differentiable at $t$ and the mean square derivative is given by

$$
\frac{\mathrm{d} X(g(t))}{\mathrm{d} t}=\left.\frac{\mathrm{d} X(v)}{\mathrm{d} v}\right|_{v=g(t)} g^{\prime}(t) .
$$

Also connected with the previous result and, as it shall be seen later, we will require to apply the mean square derivative of a random power series in order to formally construct the solution of the random fractional linear differential equation with a random initial condition. For this purpose we will use the following result:

Proposition 4. [30, p.1260] Let $\mathcal{V} \subset \mathbb{R}$ be an interval, $m \geq m_{0} \geq 0$ a non-negative integer and $\left\{U_{m}(v): v \in \mathcal{V}, m \geq m_{0}\right\}$ be a sequence of 2-SPs such that

i) $U_{m}(v)$ is mean square differentiable on $\mathcal{V}$.

ii) The mean square derivative, $U_{m}^{\prime}(v)$, is mean square continuous on $\mathcal{V}$.

iii) $U(v)=\sum_{m \geq m_{0}} U_{m}(v)$ is mean square convergent on $\mathcal{V}$.

iv) $\sum_{m \geq m_{0}} U_{m}^{\prime}(v)$ is mean square uniformly convergent on $\mathcal{V}$.

Then, the 2-SP, $U(v)$, is mean square differentiable at every $v \in \mathcal{V}$ and

$$
U^{\prime}(v)=\sum_{m \geq m_{0}} U_{m}^{\prime}(v) .
$$

Throughout this paper, $\Gamma(\alpha)$ and $\mathrm{B}\left(\alpha_{1}, \alpha_{2}\right)$ will denote the deterministic Euler gamma and beta functions, defined as

$$
\Gamma(\alpha):=\int_{0^{+}}^{\infty} \mathrm{e}^{-v} v^{\alpha-1} \mathrm{~d} v, \quad \alpha>0,
$$




$$
\mathrm{B}\left(\alpha_{1}, \alpha_{2}\right):=\int_{0}^{1} v^{\alpha_{1}-1}(1-v)^{\alpha_{2}-1} \mathrm{~d} v, \quad \alpha_{1}, \alpha_{2}>0,
$$

172

respectively. These special functions are related by the following well-known relationship

$$
\mathrm{B}\left(\alpha_{1}, \alpha_{2}\right)=\frac{\Gamma\left(\alpha_{1}\right) \Gamma\left(\alpha_{2}\right)}{\Gamma\left(\alpha_{1}+\alpha_{2}\right)}, \quad \alpha_{1}, \alpha_{2}>0 .
$$

The so-called duplication formula of the deterministic gamma function

$$
\Gamma(\alpha+1)=\alpha \Gamma(\alpha), \quad \alpha>0,
$$

will also be required later. Although these functions and relationships can be extended for $\alpha$, $\alpha_{1}$ and $\alpha_{2}$ lying in the whole complex plane except the negative integers, here they will only be applied when $\alpha>0, \alpha_{1}>0$ and $\alpha_{2}>0$. Also, the following asymptotic approximation to the gamma function will be need later [31, pp. 227]

$$
\Gamma(x+1) \approx x^{x} \mathrm{e}^{-x} \sqrt{2 \pi x}, \quad x \rightarrow+\infty .
$$

Notice that this approximation is just a generalization of the celebrated Stirling's formula.

We conclude this section by stating a technical result related to the convergence of double series that will be applied to develop the numerical examples exhibited in Section 6.

Proposition 5. [32, Lemma 9.1, ch.9] A double series $\sum_{m \geq m_{0}} \sum_{n \geq n_{0}} a_{m n}$ is absolutely convergent if and only if the following conditions hold

(i) There are $\left(m_{0}, n_{0}\right) \in \mathbb{N} \times \mathbb{N}$ and $\alpha_{0}>0$ such that

$$
\sum_{m=m_{0}}^{M} \sum_{n=n_{0}}^{N}\left|a_{m n}\right| \leq \alpha_{0} \quad \text { for all } M \geq m_{0}, N \geq n_{0}
$$

(ii) Each row-series and each column-series are absolutely convergent.

\section{Mean square random fractional differential and integral operators}

This section is addressed to introduce the random Riemann-Liouville fractional integral and the random Caputo fractional derivative in the mean square sense. As it shall see later, both random fractional operators extend their deterministic counterparts. Their definitions are based on the random mean square calculus. Firstly, we give the definition of the mean square random Riemann-Liouville fractional integral.

Definition 1. Let $\mathcal{D}=[a, b],-\infty<a<b<+\infty$, be a finite interval of the real line, $\mathbb{R}$. Let $\{X(t): t \in \mathcal{D}\}$ be a 2-SP. The random mean square (left-sided) Riemann-Liouville fractional integral of $X(t), \mathrm{J}_{a+}^{\alpha} X$, of order $\alpha>0$ is defined by

$$
\left(\mathrm{J}_{a+}^{\alpha} X\right)(t):=\frac{1}{\Gamma(\alpha)} \int_{a}^{t}(t-u)^{\alpha-1} X(u) \mathrm{d} u, \quad t \in \mathcal{D}=[a, b],
$$


Notice that the integral that appears in the right-hand side of (12) is understood in the mean square Riemann integral sense introduced in Section 1.

Remark 2. Analogously to Definition 1, we can define the random mean square (right-sided) Riemann-Liouville fractional integral of a 2-SP, $X(t)$, as

$$
\left(\mathrm{J}_{b-}^{\alpha} X\right)(t):=\frac{1}{\Gamma(\alpha)} \int_{t}^{b}(u-t)^{\alpha-1} X(u) \mathrm{d} u .
$$

Throughout this paper, the random mean square (left-sided) Riemann-Liouville fractional integral will be used only.

Keeping the notation of Definition 1, and applying Remark 1 with the following identification $d=t \in \mathcal{D}$ an arbitrary but fixed number and $g(u, d)=(d-u)^{\alpha-1} / \Gamma(\alpha)$, one deduces the following characterization of the existence of the random mean square (left-sided) Riemann-Liouville fractional integral of a $2-\operatorname{SP}\{X(t): t \in \mathcal{D}\}$.

Proposition 6. Let $\{X(t): t \in \mathcal{D}\}$ be a 2-SP with correlation function $\Gamma_{X}(\cdot, \cdot)$. Then, its random mean square (left-sided) Riemann-Liouville fractional integral, denoted by $\left(\mathbf{J}_{a+}^{\alpha} X\right)(t), \alpha>0$, exists in the mean square sense if, and only if the following deterministic double Riemann integral

$$
\int_{a}^{t} \int_{a}^{t}\left(t-u_{1}\right)^{\alpha-1}\left(t-u_{2}\right)^{\alpha-1} \Gamma_{X}\left(u_{1}, u_{2}\right) \mathrm{d} u_{1} \mathrm{~d} u_{2}
$$

exists and is finite for each $t \in \mathcal{D}$.

Now we give a sufficient condition in order to guarantee the existence of random mean square (left-sided) Riemann-Liouville fractional integral, $\left(\mathrm{J}_{a+}^{\alpha} X\right)(t)$.

Proposition 7. Let $\alpha>0$ and $\{X(t): t \in \mathcal{D}\}$ be a 2-SP such as

$$
\int_{a}^{t}(t-u)^{\alpha-1}\|X(u)\|_{2} \mathrm{~d} u<+\infty .
$$

Then, the random mean square (left-sided) Riemann-Liouville fractional integral $\left(\mathrm{J}_{a+}^{\alpha} X\right)(t)$ exists.

Proof. By Proposition 6, it is enough showing that the double deterministic integral (13) is absolutely convergent. This follows by applying inequality (5) and Fubini's theorem

$$
\begin{aligned}
& \int_{a}^{t} \int_{a}^{t}\left|\left(t-u_{1}\right)^{\alpha-1}\left(t-u_{2}\right)^{\alpha-1} \Gamma_{X}\left(u_{1}, u_{2}\right)\right| \mathrm{d} u_{1} \mathrm{~d} u_{2} \\
\leq & \int_{a}^{t} \int_{a}^{t}\left(t-u_{1}\right)^{\alpha-1}\left(t-u_{2}\right)^{\alpha-1}\left\|X\left(u_{1}\right)\right\|_{2}\left\|X\left(u_{2}\right)\right\|_{2} \mathrm{~d} u_{1} \mathrm{~d} u_{2} \\
= & \left(\int_{a}^{t}\left(t-u_{1}\right)^{\alpha-1}\left\|X\left(u_{1}\right)\right\|_{2} \mathrm{~d} s\right)\left(\int_{a}^{t}\left(t-u_{2}\right)^{\alpha-1}\left\|X\left(u_{2}\right)\right\|_{2} \mathrm{~d} u_{2}\right) \\
= & \left(\int_{a}^{t}(t-u)^{\alpha-1}\|X(u)\|_{2} \mathrm{~d} u\right)^{2}<+\infty .
\end{aligned}
$$

Notice that in the last step we have used hypothesis (14).

Apart from the fractional Riemann-Liouville integral, in the deterministic scenario it is also useful the concept of fractional derivative. In the subsequent development we introduce the definition of the random (left-sided) fractional Caputo derivative, in the mean square sense. Thus, we firstly give a characterization of its existence, and secondly, a sufficient condition in order to guarantee its existence, in the mean square sense. 
Definition 2. Let $\mathcal{D}=[a, b],-\infty<a<b<\infty$, be a finite interval of the real line $\mathbb{R}$. Let $\{X(t): t \in \mathcal{D}\}$ be a 2-SP. The random mean square (left-sided) Caputo fractional derivative of $X(t),\left({ }^{C} D_{a+}^{\alpha} X\right)(t)$, of order $\alpha>0$ is defined by

$$
\left({ }^{C} D_{a+}^{\alpha} X\right)(t):=\left(\mathrm{J}_{a+}^{n-\alpha} X^{(n)}\right)(t)=\frac{1}{\Gamma(n-\alpha)} \int_{a}^{t}(t-u)^{n-\alpha-1} X^{(n)}(u) \mathrm{d} u,
$$

where $n=-[-\alpha]$, being $[\cdot]$ the integer part function and, $X^{(n)}(t)$ denotes the $n$-th mean square derivative of the 2-SP $X(t)$.

Naturally, the integral that appears in the right-hand side of (15) is a mean square Riemann integral.

Remark 3. Analogously to Definition 2, the random mean square (right-sided) Caputo fractional derivative of a 2-SP $\{X(t): t \in \mathcal{D}=[a, b]\},-\infty<a<b<\infty$, is defined as

$$
\left({ }^{C} D_{b-}^{\alpha} X\right)(t):=\left(\mathrm{J}_{b-}^{n-\alpha} X^{(n)}\right)(t)=\frac{1}{\Gamma(n-\alpha)} \int_{t}^{b}(u-t)^{n-\alpha-1} X^{(n)}(u) \mathrm{d} u .
$$

Applying Proposition 6 to the 2-SP $X^{(n)}(t)$ and using the relationship (6), one straightforwardly gets the following characterization on the existence of the random mean square Caputo fractional derivative of a 2-SP, $X(t)$, that is $n$-times mean square differentiable.

Proposition 8. Let $\{X(t): t \in \mathcal{D}\},-\infty<a<b<\infty$, be a 2-SP n-times differentiable with correlation function $\Gamma_{X}(\cdot, \cdot)$. Then, its (left-sided) Caputo fractional derivative, $\left({ }^{C} D_{a+}^{\alpha} X\right)(t), \alpha>$ 0 , exists in the mean square sense if, and only if, the following deterministic double Riemann integral

$$
\int_{a}^{t} \int_{a}^{t}\left(t-u_{1}\right)^{n-\alpha-1}\left(t-u_{2}\right)^{n-\alpha-1} \frac{\partial^{2 n} \Gamma_{X}\left(u_{1}, u_{2}\right)}{\partial u_{1}^{n} \partial u_{2}^{n}} \mathrm{~d} u_{1} \mathrm{~d} u_{2}
$$

exists and is finite.

On the one hand, if we assume that the 2-SP $\{X(t): t \in \mathcal{D}\}$ is $n$-times mean square differentiable, then applying (5) to its $n$-th mean square derivative, $X^{(n)}(t)$, which is also a 2-SP, one gets

$$
\Gamma_{X^{(n)}}\left(u_{1}, u_{2}\right) \leq\left\|X^{(n)}\left(u_{1}\right)\right\|_{2}\left\|X^{(n)}\left(u_{2}\right)\right\|_{2} .
$$

On the other hand, using an analogous reasoning that was exhibited in the proof of Proposition 7 yields

$$
\begin{aligned}
& \int_{a}^{t} \int_{a}^{t}\left|\left(t-u_{1}\right)^{n-\alpha-1}\left(t-u_{2}\right)^{n-\alpha-1} \Gamma_{X^{(n)}}\left(u_{1}, u_{2}\right)\right| \mathrm{d} u_{1} \mathrm{~d} u_{2} \\
\leq & \int_{a}^{t} \int_{a}^{t}\left(t-u_{1}\right)^{n-\alpha-1}\left(t-u_{2}\right)^{n-\alpha-1}\left\|X^{(n)}\left(u_{1}\right)\right\|_{2}\left\|X^{(n)}\left(u_{2}\right)\right\|_{2} \mathrm{~d} u_{1} \mathrm{~d} u_{2} \\
= & \left(\int_{a}^{t}(t-u)^{n-\alpha-1}\left\|X^{(n)}(u)\right\|_{2} \mathrm{~d} u\right)^{2} .
\end{aligned}
$$

Then, the following result has been established: 
Proposition 9. Let $\alpha>0$ and $\{X(t): t \in \mathcal{D}\}$ be a 2-SP $n$-times mean square differentiable such that

$$
\int_{a}^{t}(t-u)^{n-\alpha-1}\left\|X^{(n)}(u)\right\|_{2} \mathrm{~d} u<+\infty .
$$

Then, the random (left-sided) Caputo fractional derivative, $\left({ }^{C} D_{a+}^{\alpha} X\right)(t)$, exists.

Example 1. Let $X(t)=A t^{\alpha m}, t \in[0, T], T>0,0<\alpha<1, m>0$, and assume that $A$ is $a$ bounded $R V$ (hence $A \in \mathrm{L}^{2}(\Omega)$ ). Then, $X(t)$ is a $2-S P$

$$
\left(\|X(t)\|_{2}\right)^{2}=\mathbb{E}\left[A^{2} t^{2 \alpha m}\right]=t^{2 \alpha m} \mathbb{E}\left[A^{2}\right]<+\infty,
$$

since $\mathrm{E}\left[A^{2}\right]=\left(\|A\|_{2}\right)^{2}<+\infty$. Moreover, $X(t)$ is mean square differentiable and its mean square derivative is given by $X^{\prime}(t)=\alpha m A t^{\alpha m-1}$

$$
\begin{aligned}
\left(\left\|\frac{X(t+h)-X(t)}{h}-X^{\prime}(t)\right\|_{2}\right)^{2} & =\mathbb{E}\left[\left(\frac{A(t+h)^{\alpha m}-A t^{\alpha m}}{h}-\alpha m A t^{\alpha m-1}\right)^{2}\right] \\
& =\mathbb{E}\left[A^{2}\right]\left(\frac{(t+h)^{\alpha m}-t^{\alpha m}}{h}-\alpha m t^{\alpha m-1}\right) \stackrel{h \rightarrow 0}{\longrightarrow} 0,
\end{aligned}
$$

since $\mathbb{E}\left[A^{2}\right]<+\infty$ and $g(t)=t^{\alpha m}$ is a deterministic differentiable function whose derivative is $\alpha m t^{\alpha m-1}$. Finally, according to Proposition 9 with $n=1, a=0$, we need to check the following deterministic integral

$$
\int_{0}^{t}(t-u)^{-\alpha}\left\|\alpha m A u^{\alpha m-1}\right\|_{2} \mathrm{~d} u=\alpha m\|A\|_{2} \int_{0}^{t}(t-u)^{-\alpha} u^{\alpha m-1} \mathrm{~d} u
$$

is convergent. Since $\|A\|_{2}<+\infty$, it is enough to show that the last integral is finite. To this end, let us make the change of variable: $u=v t$, then using the definition of the beta function (see (8)) and its relationship with the gamma function (see (9)), one gets

$$
\begin{aligned}
\int_{0}^{t}(t-u)^{-\alpha} u^{\alpha m-1} \mathrm{~d} u & =t^{\alpha(m-1)} \int_{0}^{1} v^{\alpha m-1}(1-v)^{-\alpha} \mathrm{d} v \\
& =t^{\alpha(m-1)} B(\alpha m, 1-\alpha) \\
& =t^{\alpha(m-1)} \frac{\Gamma(\alpha m) \Gamma(1-\alpha)}{\Gamma(\alpha(m-1)+1)}<+\infty .
\end{aligned}
$$

Moreover the value of the random mean square Caputo fractional derivative of $X(t)=A t^{\alpha m}$ is given by

$$
\left({ }^{C} D_{0^{+}}^{\alpha} X\right)(t)=\frac{1}{\Gamma(1-\alpha)} \int_{0}^{t}(t-u)^{-\alpha} \alpha m A u^{\alpha m-1} \mathrm{~d} u=\frac{\alpha m A}{\Gamma(1-\alpha)} \int_{0}^{t}(t-u)^{-\alpha} u^{\alpha m-1} \mathrm{~d} u .
$$

Observe that the commutation between the mean square integral and the RV A that we have done in the last step is legitimated because A is a bounded RV (see Proposition 1). Finally, substituting expression (16) into (17) and using property (10), one gets the following closed expression for the random (left-sided) Caputo fractional derivative of $X(t)$

$$
\left({ }^{C} D_{0^{+}}^{\alpha} X\right)(t)=\frac{\alpha m A}{\Gamma(1-\alpha)} t^{\alpha(m-1)} \int_{0}^{1} v^{\alpha m-1}(1-v)^{-\alpha} \mathrm{d} v=A \frac{\Gamma(\alpha m+1)}{\Gamma(\alpha(m-1)+1)} t^{\alpha(m-1)} .
$$




\section{Solving the random linear fractional differential equation by the mean square general-} ized Fröbenius method

This section is devoted to construct a solution SP to the random fractional linear differential initial value problem (IVP)

$$
\left\{\begin{array}{cl}
\left({ }^{C} D_{0^{+}}^{\alpha} Y\right)(t)-\lambda Y(t) & =\gamma, \quad t>0,0<\alpha \leq 1, \\
Y(0) & =\beta_{0},
\end{array}\right.
$$

where $\beta_{0}, \lambda$ and $\gamma$ are RVs defined in a common complete probability space $(\Omega, \mathcal{F}, \mathbb{P})$ satisfying certain conditions to be specified later (see hypotheses H1-H2). The solution SP will be constructed by extending the Fröbenius method to random fractional differential equations using the random Caputo fractional derivative, that we have previously introduced. The aforementioned extension will be done using the mean square random calculus.

We will seek a solution SP to the random IVP (18) of the form

$$
Y(t)=\sum_{m \geq 0} X_{m} t^{\alpha m}, \quad 0 \leq t \leq T, T>0
$$

imposing that it satisfies the random fractional differential equation. Notice that coefficients of series $Y(t)$ have been denoted by $X_{m}$ instead of $Y_{m}$. This fact will be apparent later. As expression (19) is a generalized random power series, in order to take advantage of the mean square random calculus for standard random power series

$$
X(v)=\sum_{m \geq 0} X_{m} v^{m}, \quad 0 \leq v \leq T^{\alpha}, T>0,
$$

let us consider the following expression for the random fractional derivative of the generalized random power series (19), in terms of the standard random power series (20),

$$
\left({ }^{C} D_{0^{+}}^{\alpha} Y\right)(t)=\left({ }^{C} D_{0^{+}}^{\alpha} X\right)\left(t^{\alpha}\right)=\left(\mathrm{J}_{0^{+}}^{1-\alpha} Z\right)(t),
$$

where $Z \equiv Z(t)=\left(X\left(t^{\alpha}\right)\right)^{\prime}$ denotes the mean square derivative of the SP $X(t)$ compounded with the deterministic function $t^{\alpha}$. Observe that in agreement with (15), the notation $\left(\mathrm{J}_{0^{+}}^{1-\alpha} Z\right)(t)$ in (21) stands for the the Caputo fractional derivative of $Z(t)$ with $a=0$ and $n=1$.

Let us assume that for $t$ fixed in $[0, T]$ the following conditions C1-C2 fulfill:

C1 : $X(v)$, given by (20), is a mean square differentiable at $v=t^{\alpha}$. Moreover,

$$
X^{\prime}\left(t^{\alpha}\right)=\sum_{m \geq 1} m X_{m} t^{\alpha(m-1)}
$$

$$
\text { C2 : } \frac{\mathrm{d} X(v)}{\mathrm{d} v} \text { is mean square continuous on } v \in\left[0, T^{\alpha}\right] \text {. }
$$

As $0<\alpha \leq 1$, it follows that $\mathcal{V}=\left[0, T^{\alpha}\right]$ contains the range of $g(t)=t^{\alpha}$, i.e., $g\left(\left[a_{1}, a_{2}\right]\right)=$ $g([0, T])=\left[0, T^{\alpha}\right] \subseteq\left[0, T^{\alpha}\right]$. Then, by Theorem $1 X(g(t))$ is mean square differentiable at $t$ and its mean square derivative is given by

$$
Z(t):=Y^{\prime}(t)=\left(X\left(t^{\alpha}\right)\right)^{\prime}=\alpha t^{\alpha-1} X^{\prime}\left(t^{\alpha}\right) .
$$




$$
\begin{aligned}
\left({ }^{C} D_{0^{+}}^{\alpha} Y\right)(t) & =\left(\mathrm{J}_{0^{+}}^{1-\alpha} Z\right)(t) \\
& =\frac{1}{\Gamma(1-\alpha)} \int_{0^{+}}^{t}(t-u)^{-\alpha}\left(\alpha u^{\alpha-1} X^{\prime}\left(u^{\alpha}\right)\right) \mathrm{d} u \\
& =\frac{1}{\Gamma(1-\alpha)} \int_{0^{+}}^{t}(t-u)^{-\alpha}\left(\alpha u^{\alpha-1} \sum_{m \geq 1} m X_{m} u^{\alpha(m-1)}\right) \mathrm{d} u \\
& =\frac{1}{\Gamma(1-\alpha)} \int_{0^{+}}^{t}(t-u)^{-\alpha}\left(\sum_{m \geq 1} \alpha m X_{m} u^{\alpha m-1}\right) \mathrm{d} u .
\end{aligned}
$$

We will further assume that the following condition is satisfied

C3 : The random generalized power series $\sum_{m \geq 1} m X_{m} t^{\alpha m-1}$ is mean square uniformly convergent on the domain $0 \leq t \leq T$.

Then, the integral and the infinite sum that appear in (24) can be commuted, and applying the results shown in Example 1, expression (24) can be written as

$$
\begin{aligned}
\left({ }^{C} D_{0^{+}}^{\alpha} Y\right)(t) & =\frac{1}{\Gamma(1-\alpha)} \sum_{m \geq 1}\left(\alpha m X_{m} \int_{0^{+}}^{t}(t-u)^{-\alpha} u^{\alpha m-1} \mathrm{~d} u\right) \\
& =\sum_{m \geq 1}\left(X_{m} \frac{\Gamma(\alpha m+1)}{\Gamma(\alpha(m-1)+1)} t^{\alpha(m-1)}\right) \\
& =\sum_{m \geq 0}\left(X_{m+1} \frac{\Gamma(\alpha(m+1)+1)}{\Gamma(\alpha m+1)} t^{\alpha m}\right) .
\end{aligned}
$$

It is important to point out that conditions C1-C3 will be checked once the RVs $X_{m}$, that define the random power series (20), are determined for the random IVP (18). With this goal and using the Fröbenius method, we impose that the random generalized power series solution (19) satisfies the random fractional differential equation given in (18). Substituting formally expressions (19) and (25) into (18), one gets

$$
\sum_{m \geq 0}\left(X_{m+1} \frac{\Gamma(\alpha(m+1)+1)}{\Gamma(\alpha m+1)} t^{\alpha m}\right)-\lambda \sum_{m \geq 0} X_{m} t^{\alpha m}=\gamma,
$$

$$
\sum_{m \geq 0}\left(X_{m+1} \frac{\Gamma(\alpha(m+1)+1)}{\Gamma(\alpha m+1)}-\lambda X_{m}\right) t^{\alpha m}-\gamma=0
$$

$$
X_{1} \Gamma(\alpha+1)-\lambda X_{0}-\gamma+\sum_{m \geq 1}\left(X_{m+1} \frac{\Gamma(\alpha(m+1)+1)}{\Gamma(\alpha m+1)}-\lambda X_{m}\right) t^{\alpha m}=0 .
$$

Therefore, a candidate solution SP of the form (19) to the random IVP (18) can be constructed if the coefficients $X_{m}$ are chosen so that they satisfy the following recurrence

$$
X_{1}=\frac{\lambda \beta_{0}+\gamma}{\Gamma(\alpha+1)}, \quad X_{m+1}=\frac{\lambda \Gamma(\alpha m+1)}{\Gamma(\alpha(m+1)+1)} X_{m}, \quad m \geq 1,
$$


where, we have used that the initial condition is $Y(0)=X_{0}=\beta_{0}$. The recursive application of this relationship yields

$$
X_{m}=\frac{\lambda^{m} \beta_{0}+\lambda^{m-1} \gamma}{\Gamma(\alpha m+1)}, \quad m \geq 1, \quad X_{0}=\beta_{0} .
$$

Summarizing, a candidate random generalized power series solution to the IVP (18) is given by

$$
Y(t)=X\left(t^{\alpha}\right), \quad X(v)=\sum_{m \geq 0} X_{m, 1} v^{m}+\sum_{m \geq 1} X_{m, 2} v^{m}, \text { where }\left\{\begin{aligned}
X_{m, 1} & =\frac{\lambda^{m} \beta_{0}}{\Gamma(\alpha m+1)}, \\
X_{m, 2} & =\frac{\lambda^{m-1} \gamma}{\Gamma(\alpha m+1)},
\end{aligned}\right.
$$

that is,

$$
Y(t)=\sum_{m \geq 0} \frac{\lambda^{m} \beta_{0}}{\Gamma(\alpha m+1)} t^{\alpha m}+\sum_{m \geq 1} \frac{\lambda^{m-1} \gamma}{\Gamma(\alpha m+1)} t^{\alpha m} .
$$

Remark 4. The so-called Mittag-Leffler function

$$
E_{\alpha, v}(z)=\sum_{m \geq 0} \frac{z^{m}}{\Gamma(\alpha m+v)}, \quad z \in \mathbb{R}, \alpha, v \geq 0,
$$

plays a key role in the investigation of deterministic fractional differential equations. Looking at the expression (27), which is a random generalized power series, it is suggested a strong connection with the Mittag-Leffler function and the solution found using the random generalized Fröbenius technique, namely,

$$
Y(t)=\beta_{0} E_{\alpha, 1}\left(\lambda t^{\alpha}\right)+\gamma t^{\alpha} E_{\alpha, \alpha+1}\left(\lambda t^{\alpha}\right) .
$$

Notice that the study previously performed provides sufficient conditions on the RV $\lambda$ in order to extend the Mittag-Leffler function to the random framework since it is well-defined in the Banach space $\left(\mathrm{L}^{2}(\Omega),\|\cdot\|_{2}\right)$ introduced in (2).

So far, we have formally constructed a random generalized power series solution to the random IVP (18), which is given by (27). Henceforth, we will prove that it is really a rigorous solution by checking that conditions $\mathbf{C 1}-\mathbf{C} 3$ hold. This will be done for a rich enough class of RVs, denoted by $\mathfrak{C}$, that contains significant RVs and that enables us to construct accurate approximations for another important RVs that do not belong to the class $\mathfrak{C}$. These issues will be discussed later.

Definition 3. $A R V, X$, is said to belong to the class $\mathfrak{C}$ if, and only if, there exist positive constants $L>0$ and $H>0$ such that

$$
\mathbb{E}\left[|X|^{m}\right] \leq L H^{m}<+\infty, \quad \forall m \geq 0 .
$$

Remark 5. Notice that condition (29) is equivalent to

$$
\mathbb{E}\left[|X|^{m}\right]=O\left(H^{m}\right), \quad H>0, m \geq 0,
$$

22 where $O(\cdot)$ stands for the Landau symbol. Observe that every RV of class $\mathbb{C}$ is a 2-RV. 
Remark 6. It is important to point out that this class of RVs has already been used successfully to deal with the analysis of some classical (non-fractional) random differential equations [33, 34] As it is shown in [33], bounded RVs belong to the class $\mathfrak{C}$. Hence, relevant RVs such as binomial, hypergeometric, uniform, trapezoidal, beta, $\lambda$-distributed, etc., are of class $\mathfrak{C}$. While important unbounded RVs like Poisson, exponential, gaussian, etc. can be approximated by truncating appropriately their domain, that is, using bounded RVs.

Now, we are going to legitimate the conditions C1-C3 we have imposed to formally construct the random generalized power solution (27). Hereinafter, we will assume the following hypotheses:

H1 : The input data $\beta_{0}, \gamma$ and $\lambda$ are independent 2-RVs.

H2 : $\lambda$ belongs to the class $\mathfrak{C}$ introduced in Definition 3.

Observe that hypothesis $\mathbf{H} \mathbf{2}$ entails that

$$
\left\|\beta_{0}\right\|_{2}<+\infty, \quad\|\gamma\|_{2}<+\infty, \quad\left\|\lambda^{m}\right\|_{2}<\sqrt{L} H^{m}<+\infty, \quad \forall m \geq 0,
$$

being $L$ and $H$ the positive constants introduced in Definition 3. The above bound for $\lambda^{m}$ follows from the definition of the $\|\cdot\|_{2}$-norm given in (2) and (30)

$$
\left\|\lambda^{m}\right\|_{2}=\sqrt{\mathbb{E}\left[|\lambda|^{2 m}\right]} \leq \sqrt{L H^{2 m}}=\sqrt{L} H^{m}<+\infty, \quad \forall m \geq 0 .
$$

To check condition C1 we will apply Proposition 4 to the two series defined in (26). Specifically, for the first series in (26) we apply Proposition 4 with the following identification: $m_{0}=0$, $U_{m}(v)=X_{m, 1} v^{m}$. Firstly, we prove that, for each $m \geq 0$ fixed, $X_{m, 1}(v):=X_{m, 1} v^{m}=\left(\lambda^{m} \beta_{0}\right) /(\Gamma(\alpha m+$ 1)) $v^{m}$ is mean square differentiable at $v=t^{\alpha}$, being $X_{m, 1}^{\prime}\left(t^{\alpha}\right)=m \lambda^{m} \beta_{0} v^{\alpha(m-1)} / \Gamma(\alpha m+1)$ its is mean square derivative. Indeed, observe that for every $v$ such that $0<v \leq T, T>0$, one gets

$$
\begin{aligned}
0 & <\left\|\frac{X_{m, 1}\left(t^{\alpha}+h\right)-X_{m, 1}\left(t^{\alpha}\right)}{h}-X_{m, 1}^{\prime}\left(t^{\alpha}\right)\right\|_{2} \\
& =\left\|\frac{\frac{\lambda^{m} \beta_{0}}{\Gamma(\alpha m+1)}\left(t^{\alpha}+h\right)^{m}-\frac{\lambda^{m} \beta_{0}}{\Gamma(\alpha m+1)} t^{\alpha m}}{h}-\frac{m \lambda^{m} \beta_{0}}{\Gamma(\alpha m+1)} t^{\alpha(m-1)}\right\|_{2} \\
& =\left\|\frac{\lambda^{m} \beta_{0}}{\Gamma(\alpha m+1)}\left(\frac{\left(t^{\alpha}+h\right)^{m}-t^{\alpha m}}{h}-m t^{\alpha(m-1)}\right)\right\|_{2} \\
& =\left\|\lambda^{m} \beta_{0}\right\|_{2} \frac{1}{\Gamma(\alpha m+1)}\left|\frac{\left(t^{\alpha}+h\right)^{m}-t^{\alpha m}}{h}-m t^{\alpha(m-1)}\right| \\
& \stackrel{(\mathrm{I})}{=}\left\|\lambda^{m}\right\|_{2}\left\|\beta_{0}\right\|_{2} \frac{1}{\Gamma(\alpha m+1)}\left|\frac{\left(t^{\alpha}+h\right)^{m}-t^{\alpha m}}{h}-m t^{\alpha(m-1)}\right| \\
& \stackrel{(\mathrm{II})}{\leq} \sqrt{L} H^{m}\left\|\beta_{0}\right\|_{2} \frac{1}{\Gamma(\alpha m+1)}\left|\frac{\left(t^{\alpha}+h\right)^{m}-t^{\alpha m}}{h}-m t^{\alpha(m-1)}\right| \underset{h \rightarrow 0}{\longrightarrow} 0,
\end{aligned}
$$

where in the step (I) we have applied the hypothesis $\mathbf{H 1}$ of statistical independence of RVs $\beta_{0}$ and $\lambda$ together with Proposition 2 using the definition of the $\|\cdot\|_{2}$-norm in terms of the expectation operator; in step (II) we have directly used (31) and, finally for the last limit we have used that 
the deterministic function $h(v)=v^{m}$ is differentiable at $v=t^{\alpha}$ and that $\beta_{0}$ is a 2-RV, hence $\left\|\beta_{0}\right\|_{2}<+\infty$.

Secondly, we need to prove that for each $m \geq 1$ fixed, $X_{m, 1}^{\prime}(v)=m \lambda^{m} \beta_{0} v^{m-1} / \Gamma(\alpha m+1)$ is mean square continuous at $v=t^{\alpha}$. This can be checked by following an analogous reasoning to the one exhibited in (32)

$$
\begin{aligned}
0 & <\left\|X_{m, 1}^{\prime}\left(t^{\alpha}+h\right)-X_{m, 1}^{\prime}\left(t^{\alpha}\right)\right\|_{2} \\
& =\left\|\frac{m \lambda^{m} \beta_{0}\left(t^{\alpha}+h\right)^{m-1}}{\Gamma(\alpha m+1)}-\frac{m \lambda^{m} \beta_{0} t^{\alpha(m-1)}}{\Gamma(\alpha m+1)}\right\|_{2} \\
& =\left\|\frac{m \lambda^{m} \beta_{0}}{\Gamma(\alpha m+1)}\left(\left(t^{\alpha}+h\right)^{m-1}-t^{\alpha(m-1)}\right)\right\|_{2} \\
& =\left\|\lambda^{m} \beta_{0}\right\|_{2} \frac{m}{\Gamma(\alpha m+1)}\left|\left(t^{\alpha}+h\right)^{m-1}-t^{\alpha(m-1)}\right| \\
& =\left\|\lambda^{m}\right\|_{2}\left\|\beta_{0}\right\|_{2} \frac{m}{\Gamma(\alpha m+1)}\left|\left(t^{\alpha}+h\right)^{m-1}-t^{\alpha(m-1)}\right| \\
& \leq \sqrt{L} H^{m}\left\|\beta_{0}\right\|_{2} \frac{\alpha m}{\Gamma(\alpha m+1)}\left|\left(t^{\alpha}+h\right)^{m-1}-t^{\alpha(m-1)}\right| \underset{h \rightarrow 0}{\longrightarrow} 0, \quad t \leq T, T>0,
\end{aligned}
$$

where in the last step we have applied that the deterministic function $v^{m-1}$ is continuous at $t^{\alpha}$. Thirdly, we shall check that the random power series $\sum_{m \geq 0} X_{m, 1}(v)=\sum_{m \geq 0} X_{m, 1} v^{m}$ is mean square convergent for every $v: 0<v \leq T^{\alpha}$. To do that we will majorize the deterministic series $\sum_{m \geq 0}\left\|X_{m, 1}(v)\right\|_{2}=\sum_{m \geq 0}\left\|X_{m, 1}\right\|_{2} v^{m}$ by a convergent series. With this goal, let us observe that

$$
\left\|X_{m, 1}\right\|_{2} v^{m}=\left\|\frac{\lambda^{m} \beta_{0}}{\Gamma(\alpha m+1)}\right\|_{2} v^{m} \leq \sqrt{L} H^{m}\left\|\beta_{0}\right\|_{2} \frac{v^{m}}{\Gamma(\alpha m+1)}:=\delta_{m}(v), \quad 0<v \leq T^{\alpha}, T>0 .
$$

Then, using the test ratio for numerical series together with the asymptotic approximation of the gamma function given in (11), one gets

$$
\begin{aligned}
\lim _{m \rightarrow+\infty} \frac{\delta_{m+1}(v)}{\delta_{m}(v)} & =H\left(\lim _{m \rightarrow+\infty} \frac{\Gamma(\alpha m+1)}{\Gamma(\alpha(m+1)+1)}\right) v \\
& =H\left(\lim _{m \rightarrow+\infty} \frac{(\alpha m)^{\alpha m} \mathrm{e}^{-\alpha m} \sqrt{2 \pi \alpha m}}{(\alpha(m+1))^{\alpha(m+1)} \mathrm{e}^{-\alpha(m+1)} \sqrt{2 \pi \alpha(m+1)}}\right) v \\
& =H\left(\lim _{m \rightarrow+\infty}\left(\frac{m}{m+1}\right)^{\alpha m} \frac{1}{(\alpha(m+1))^{\alpha}} \sqrt{\frac{m}{m+1}}\right) \mathrm{e}^{\alpha} v=0 \\
& =H\left(\lim _{m \rightarrow+\infty} \frac{1}{(\alpha(m+1))^{\alpha}} \sqrt{\frac{m}{m+1}}\right) v=0,
\end{aligned}
$$

where we have used that

$$
\lim _{m \rightarrow+\infty}\left(\frac{m}{m+1}\right)^{\alpha m}=\lim _{m \rightarrow+\infty}\left(\frac{1}{1+\frac{1}{m}}\right)^{\alpha m}=\lim _{m \rightarrow+\infty}\left(1+\frac{1}{m}\right)^{-\alpha m}=\mathrm{e}^{-\alpha} .
$$

This proves the mean square convergence of the random power series $\sum_{m \geq 0} X_{m, 1} v^{m}$ defined in (26) for every $v$ in $0<v \leq T^{\alpha}$. Fourthly, we shall prove the mean square uniform convergence of the random power series $\sum_{m \geq 0} X_{m, 1}^{\prime}(v)=\sum_{m \geq 1} m X_{m, 1} v^{m-1}$, being $X_{m, 1}=\lambda^{m} \beta_{0} / \Gamma(\alpha m+1)$ on 
the domain $0<v \leq T^{\alpha}, T>0$. As the reasoning is analogous to the one exhibited in (33)-(34), we just show it directly

$$
\left\|m X_{m, 1}\right\|_{2} v^{m-1}=m\left\|\frac{\lambda^{m} \beta_{0}}{\Gamma(\alpha m+1)}\right\|_{2} v^{m-1} \leq m \sqrt{L} H^{m}\left\|\beta_{0}\right\|_{2} \frac{T^{m-1}}{\Gamma(\alpha m+1)}:=\hat{\delta}_{m},
$$

and

$$
\lim _{m \rightarrow+\infty} \frac{\hat{\delta}_{m+1}}{\hat{\delta}_{m}}=H\left(\lim _{m \rightarrow+\infty} \frac{1}{(\alpha(m+1))^{\alpha}} \sqrt{\frac{m+1}{m}}\right) T=0 .
$$

All this justifies that the random power series $\sum_{m \geq 0} X_{m, 1} v^{m}$ is mean square differentiable at $v=t^{\alpha}$. By using similar arguments, one can prove that the second power series $\sum_{m>1} X_{m, 2} t^{m}$ in (26) is mean square differentiable at $v=t^{\alpha}$. Both conclusions allows us to affirm that the random power series $X(v)$, defined in (26), satisfies condition C1. As a consequence, by applying Proposition 4 the mean square derivative (22) assumed in $\mathbf{C 1}$ is legitimated. Based upon similar arguments, it can be shown that $X(v)$ also satisfies conditions $\mathbf{C 2}$ and $\mathbf{C 3}$.

Summarizing, the following result has been proved

Theorem 2. Let us consider the random fractional linear differential initial value problem (IVP)

$$
\left\{\begin{array}{cl}
\left({ }^{C} D_{0^{+}}^{\alpha} Y\right)(t)-\lambda Y(t) & =\gamma, \quad t>0,0<\alpha \leq 1, \\
Y(0) & =\beta_{0},
\end{array}\right.
$$

where the input data satisfy the following hypotheses:

H1 : The input data $\beta_{0}, \gamma$ and $\lambda$ are independent RVs.

$\boldsymbol{H} 2$ : The input data $\beta_{0}, \gamma$ and $\lambda$ are $2-R V s$, and there exist positive constants $L>0$ and $H>0$ such that

$$
\mathbb{E}\left[|\lambda|^{m}\right] \leq L H^{m}<+\infty, \quad \forall m \geq 0 .
$$

Then,

$$
Y(t)=\sum_{m \geq 0} \frac{\lambda^{m} \beta_{0}}{\Gamma(\alpha m+1)} t^{\alpha m}+\sum_{m \geq 1} \frac{\lambda^{m-1} \gamma}{\Gamma(\alpha m+1)} t^{\alpha m},
$$

is a mean square solution to the IVP that converges for all $t>0$.

\section{Computing approximations of the mean, the variance, the covariance and the cross-} covariance functions of the solution stochastic process

So far we have provided sufficient conditions in order to guarantee the mean square convergence of the solution SP defined by the random generalized power series (27). However, from a practical point of view this infinite series needs to be truncated to keep computationally feasible. This motivates the consideration of following finite sum (see (26)-(27))

$$
Y_{M}(t)=\sum_{m=0}^{M} X_{m, 1} t^{\alpha m}+\sum_{m=1}^{M} X_{m, 2} t^{\alpha m}=\sum_{m=0}^{M} \frac{\lambda^{m} \beta_{0}}{\Gamma(\alpha m+1)} t^{\alpha m}+\sum_{m=1}^{M} \frac{\lambda^{m-1} \gamma}{\Gamma(\alpha m+1)} t^{\alpha m} .
$$

From this expression we will compute approximations of both the mean and variance/standard deviation functions of the solution SP given in (27). The following property of the mean square convergence will play a key role to legitimate the computation of approximations. 
Proposition 10. $[8, p$.$] Let \left\{X_{M}: M \geq 0\right\}$ and $\left\{Z_{N}: N \geq 0\right\}$ be two sequences of 2-RVs such that $X_{M} \underset{M \rightarrow+\infty}{\stackrel{m . s .}{\rightarrow}} X$ and $Z_{N} \underset{N \rightarrow+\infty}{\stackrel{m . s .}{\rightarrow}}$ Z. Then

$$
\mathbb{E}\left[X_{M} Z_{N}\right] \underset{M, N \rightarrow+\infty}{\longrightarrow} \mathbb{E}[X Z]
$$

Firstly, let us observe that taking $t \in \mathbb{R}$ arbitrary but fixed, and using the following identification $X_{M} \equiv Y_{M}(t)$ for all $M \geq 0$, being $Y_{M}(t)$ the partial sum defined in (35) and $Z_{N} \equiv 1$ for all $N \geq 0$ in Proposition 10, then one deduces

$$
\mathbb{E}\left[Y_{M}(t)\right] \underset{M \rightarrow+\infty}{\longrightarrow} \mathbb{E}[Y(t)]
$$

since we have proved the mean square convergence of $Y_{M}(t)$ for every $t \in \mathbb{R}$. Likewise, applying Proposition 10 with $M \equiv N$ and $X_{M}=Z_{N} \equiv Y_{M}(t)$ for all $M, N \geq 0$, being $Y_{M}(t)$ the partial sum defined in (35), and taking into account that $\mathbb{V}\left[Y_{M}(t)\right]=\mathbb{E}\left[\left(Y_{M}(t)\right)^{2}\right]-\left(\mathbb{E}\left[Y_{M}(t)\right]\right)^{2}$ together with (36), one gets

$$
\mathbb{V}\left[Y_{M}(t)\right] \underset{M \rightarrow+\infty}{\longrightarrow} \mathbb{V}[Y(t)]
$$

Expressions (36) and (37) legitimize that the approximations $\mathbb{E}\left[Y_{M}(t)\right]$ and $\mathbb{V}\left[Y_{M}(t)\right]$ of the mean and the variance, respectively, constructed by $Y_{M}(t)$ given in (35) will converge to the corresponding exact values. At this point, we want to emphasize that this distinctive property of mean square convergence is what has really justified the use of this strong type of convergence in our study against alternative stochastic convergences like almost surely convergence, convergence in probability and convergence in distribution, which do not have such key property. Below, we shall provide expressions for $\mathbb{E}\left[Y_{M}(t)\right]$ and $\mathbb{V}\left[Y_{M}(t)\right]$. With this goal, let us take the expectation operator and using its linearity property together with the hypothesis $\mathbf{H 1}$ of independence for the input RVs $\beta_{0}, \gamma$ and $\lambda$ and Proposition 2, one gets

$$
\mathbb{E}\left[Y_{M}(t)\right]=\mathbb{E}\left[\beta_{0}\right] \sum_{m=0}^{M} \frac{\mathbb{E}\left[\lambda^{m}\right]}{\Gamma(\alpha m+1)} t^{\alpha m}+\mathbb{E}[\gamma] \sum_{m=1}^{M} \frac{\mathbb{E}\left[\lambda^{m-1}\right]}{\Gamma(\alpha m+1)} t^{\alpha m} .
$$

As

$$
\mathbb{V}\left[Y_{M}(t)\right]=\mathbb{E}\left[\left(Y_{M}(t)\right)^{2}\right]-\left(\mathbb{E}\left[Y_{M}(t)\right]\right)^{2},
$$

in order to compute the variance of (35) it is enough to determine an expression of $\mathbb{E}\left[\left(Y_{M}(t)\right)^{2}\right]$ in terms of the statistical moments of the input RVs $\beta_{0}, \gamma$ and $\lambda$. To achieve this goal, let us consider 
the following development

$$
\begin{aligned}
\mathbb{E}\left[\left(Y_{M}(t)\right)^{2}\right]= & \mathbb{E}\left[\left(\sum_{m=0}^{M} \frac{\lambda^{m} \beta_{0}}{\Gamma(\alpha m+1)} t^{\alpha m}+\sum_{m=1}^{M} \frac{\lambda^{m-1} \gamma}{\Gamma(\alpha m+1)} t^{\alpha m}\right)^{2}\right] \\
= & \mathbb{E}\left[\left(\sum_{m=0}^{M} \frac{\lambda^{m} \beta_{0}}{\Gamma(\alpha m+1)} t^{\alpha m}\right)^{2}\right]+\mathbb{E}\left[\left(\sum_{m=1}^{M} \frac{\lambda^{m-1} \gamma}{\Gamma(\alpha m+1)} t^{\alpha m}\right)^{2}\right] \\
& +2 \mathbb{E}\left[\left(\sum_{m=0}^{M} \frac{\lambda^{m} \beta_{0}}{\Gamma(\alpha m+1)} t^{\alpha m}\right)\left(\sum_{m=1}^{M} \frac{\lambda^{m-1} \gamma}{\Gamma(\alpha m+1)} t^{\alpha m}\right)\right] \\
= & \mathbb{E}\left[\left(\beta_{0}\right)^{2}\right]\left(\sum_{m=0}^{M} \frac{\mathbb{E}\left[\lambda^{2 m}\right]}{\Gamma^{2}(\alpha m+1)} t^{2 \alpha m}+2 \sum_{m=1}^{M} \sum_{n=0}^{m-1} \frac{\mathbb{E}\left[\lambda^{m+n}\right]}{\Gamma(\alpha m+1) \Gamma(\alpha n+1)} t^{\alpha(m+n)}\right) \\
& +\mathbb{E}\left[\gamma^{2}\right]\left(\sum_{m=1}^{M} \frac{\mathbb{E}\left[\lambda^{2(m-1)}\right]}{\Gamma^{2}(\alpha m+1)} t^{2 \alpha m}+2 \sum_{m=2}^{M} \sum_{n=1}^{m-1} \frac{\mathbb{E}\left[\lambda^{m+n-2}\right]}{\Gamma(\alpha m+1) \Gamma(\alpha n+1)} t^{\alpha(m+n)}\right) \\
& +2 \mathbb{E}\left[\beta_{0}\right] \mathbb{E}[\gamma] \sum_{m=0}^{M} \sum_{n=1}^{M} \frac{\mathbb{E}\left[\lambda^{m+n-1}\right]}{\Gamma(\alpha m+1) \Gamma(\alpha n+1)} t^{\alpha(m+n)},
\end{aligned}
$$

where the hypothesis $\mathbf{H 1}$ has been applied.

If we choose the input RVs $\beta_{0}, \gamma$ and $\lambda$ satisfying the hypotheses H1-H2, then since we have proved the unconditional mean square convergence over the whole real line of the random generalized power series SP (27), it is guaranteed that the approximations of the mean and the variance of the solution SP, $Y(t)$, to the random IVP (18), given by (38)-(39), will converge to the corresponding exact values for every $t \in \mathbb{R}$.

We finish this section by giving further probabilistic properties of the solution SP, $Y(t)$. These properties will also be constructed from the truncated series (35). First, we will calculate an approximation of the cross-covariance function of the solution SP. With this aim let us consider $M, N \geq 1, t, s \in \mathbb{R}$ and the following development based on the properties of the cross-covariance operator together with the expression (35)

$$
\begin{aligned}
\mathbb{C}_{Y_{M}, Y_{N}}(t, s)= & \operatorname{Cov}\left[Y_{M}(t), Y_{N}(s)\right] \\
= & \operatorname{Cov}\left[\sum_{m=0}^{M} X_{m, 1} t^{\alpha m}+\sum_{m=1}^{M} X_{m, 2} t^{\alpha m}, \sum_{n=0}^{N} X_{n, 1} s^{\alpha n}+\sum_{n=1}^{N} X_{n, 2} s^{\alpha n}\right] \\
= & \sum_{m=0}^{M} \sum_{n=0}^{N} \operatorname{Cov}\left[X_{m, 1}, X_{n, 1}\right] t^{\alpha m} s^{\alpha n}+\sum_{m=0}^{M} \sum_{n=1}^{N} \mathbb{C o v}\left[X_{m, 1}, X_{n, 2}\right] t^{\alpha m} s^{\alpha n} \\
& +\sum_{m=1}^{M} \sum_{n=0}^{N} \mathbb{C o v}\left[X_{m, 2}, X_{n, 1}\right] t^{\alpha m} s^{\alpha n}+\sum_{m=1}^{M} \sum_{n=1}^{N} \mathbb{C o v}\left[X_{m, 2}, X_{n, 2}\right] t^{\alpha m} s^{\alpha n},
\end{aligned}
$$

where each one of the four covariances that appear in the last double sum can be computed in terms of the input data. For example, taking into account (26), the hypothesis $\mathbf{H 1}$ of independence of RVs $\beta_{0}, \gamma$, and $\lambda$, one gets 


$$
\begin{aligned}
\operatorname{Cov}\left[X_{m, 1}, X_{n, 1}\right] & =\mathbb{E}\left[X_{m, 1} X_{n, 1}\right]-\mathbb{E}\left[X_{m, 1}\right] \mathbb{E}\left[X_{n, 1}\right] \\
& =\frac{\mathbb{E}\left[\lambda^{m+n}\left(\beta_{0}\right)^{2}\right]}{\Gamma(\alpha m+1) \Gamma(\alpha n+1)}-\frac{\mathbb{E}\left[\lambda^{m} \beta_{0}\right]}{\Gamma(\alpha m+1)} \frac{\mathbb{E}\left[\lambda^{n} \beta_{0}\right]}{\Gamma(\alpha n+1)} \\
& =\frac{\mathbb{E}\left[\lambda^{m+n}\right] \mathbb{E}\left[\left(\beta_{0}\right)^{2}\right]-\mathbb{E}\left[\lambda^{m}\right] \mathbb{E}\left[\lambda^{n}\right]\left(\mathbb{E}\left[\beta_{0}\right]\right)^{2}}{\Gamma(\alpha m+1) \Gamma(\alpha n+1)} .
\end{aligned}
$$

422

423

424 and

$$
\operatorname{Cov}\left[X_{m, 1}, X_{n, 2}\right]=\frac{\left(\mathbb{E}\left[\lambda^{m+n-1}\right]-\mathbb{E}\left[\lambda^{m}\right] \mathbb{E}\left[\lambda^{n-1}\right]\right) \mathbb{E}\left[\beta_{0}\right] \mathbb{E}[\gamma]}{\Gamma(\alpha m+1) \Gamma(\alpha n+1)},
$$

$$
\operatorname{Cov}\left[X_{m, 2}, X_{n, 1}\right]=\frac{\left(\mathbb{E}\left[\lambda^{m+n-1}\right]-\mathbb{E}\left[\lambda^{m-1}\right] \mathbb{E}\left[\lambda^{n}\right]\right) \mathbb{E}\left[\beta_{0}\right] \mathbb{E}[\gamma]}{\Gamma(\alpha m+1) \Gamma(\alpha n+1)},
$$

$$
\operatorname{Cov}\left[X_{m, 2}, X_{n, 2}\right]=\frac{\mathbb{E}\left[\lambda^{m+n-2}\right] \mathbb{E}\left[\gamma^{2}\right]-\mathbb{E}\left[\lambda^{m-1}\right] \mathbb{E}\left[\lambda^{n-1}\right](\mathbb{E}[\gamma])^{2}}{\Gamma(\alpha m+1) \Gamma(\alpha n+1)} .
$$

In an analogous manner, it can be seen that

Particularizing the expression $\mathbb{C}_{Y_{M}, Y_{N}}(t, s)$ when

- $s=t$, one obtains the covariance of the random approximations of order $M$ and $N$ at the time instant $t$ of the solution SP.

- $M=N$, one obtains the covariance of the random approximation of order $M$ at the two time instants $t$ and $s$ of the solution SP.

- $M=N$ and $s=t$, one obtains the variance of the random approximation of order $M$ at the time instant $t$ of the solution SP. This expression is equivalent to the one determined by 
(38)-(39). Specifically,

$$
\begin{aligned}
& \mathbb{V}\left[Y_{M}(t)\right]=\mathbb{C}_{Y_{M}, Y_{M}}(t, t) \\
& =\sum_{m=0}^{M} \sum_{n=0}^{M} \frac{\mathbb{E}\left[\lambda^{m+n}\right] \mathbb{E}\left[\left(\beta_{0}\right)^{2}\right]-\mathbb{E}\left[\lambda^{m}\right] \mathbb{E}\left[\lambda^{n}\right]\left(\mathbb{E}\left[\beta_{0}\right]\right)^{2}}{\Gamma(\alpha m+1) \Gamma(\alpha n+1)} t^{\alpha(m+n)} \\
& +\sum_{m=0}^{M} \sum_{n=1}^{M} \frac{\left(\mathbb{E}\left[\lambda^{m+n-1}\right]-\mathbb{E}\left[\lambda^{m}\right] \mathbb{E}\left[\lambda^{n-1}\right]\right) \mathbb{E}\left[\beta_{0}\right] \mathbb{E}[\gamma]}{\Gamma(\alpha m+1) \Gamma(\alpha n+1)} t^{\alpha(m+n)} \\
& +\sum_{m=1}^{M} \sum_{n=0}^{M} \frac{\left(\mathbb{E}\left[\lambda^{m+n-1}\right]-\mathbb{E}\left[\lambda^{m-1}\right] \mathbb{E}\left[\lambda^{n}\right]\right) \mathbb{E}\left[\beta_{0}\right] \mathbb{E}[\gamma]}{\Gamma(\alpha m+1) \Gamma(\alpha n+1)} t^{\alpha(m+n)} \\
& +\sum_{m=1}^{M} \sum_{n=1}^{M} \frac{\mathbb{E}\left[\lambda^{m+n-2}\right] \mathbb{E}\left[\gamma^{2}\right]-\mathbb{E}\left[\lambda^{m-1}\right] \mathbb{E}\left[\lambda^{n-1}\right](\mathbb{E}[\gamma])^{2}}{\Gamma(\alpha m+1) \Gamma(\alpha n+1)} t^{\alpha(m+n)} \\
& =\mathbb{E}\left[\left(\beta_{0}\right)^{2}\right] \sum_{m=0}^{M} \sum_{n=0}^{M} \frac{\mathbb{E}\left[\lambda^{m+n}\right]}{\Gamma(\alpha m+1) \Gamma(\alpha n+1)} t^{\alpha(m+n)} \\
& -\left(\mathbb{E}\left[\beta_{0}\right]\right)^{2}\left(\sum_{m=0}^{M} \frac{\mathbb{E}\left[\lambda^{m}\right]}{\Gamma(\alpha m+1)} t^{\alpha m}\right)\left(\sum_{n=0}^{M} \frac{\mathbb{E}\left[\lambda^{n}\right]}{\Gamma(\alpha n+1)} t^{\alpha n}\right) \\
& +\mathbb{E}\left[\beta_{0}\right] \mathbb{E}[\gamma] \sum_{m=0}^{M} \sum_{n=1}^{M} \frac{\left(\mathbb{E}\left[\lambda^{m+n-1}\right]\right)}{\Gamma(\alpha m+1) \Gamma(\alpha n+1)} t^{\alpha(m+n)} \\
& -\mathbb{E}\left[\beta_{0}\right] \mathbb{E}[\gamma]\left(\sum_{m=0}^{M} \frac{\mathbb{E}\left[\lambda^{m}\right]}{\Gamma(\alpha m+1)} t^{\alpha m}\right)\left(\sum_{n=1}^{M} \frac{\mathbb{E}\left[\lambda^{n-1}\right]}{\Gamma(\alpha n+1)} t^{\alpha n}\right) \\
& +\mathbb{E}\left[\beta_{0}\right] \mathbb{E}[\gamma] \sum_{m=1}^{M} \sum_{n=0}^{M} \frac{\mathbb{E}\left[\lambda^{m+n-1}\right]}{\Gamma(\alpha m+1) \Gamma(\alpha n+1)} t^{\alpha(m+n)} \\
& -\mathbb{E}\left[\beta_{0}\right] \mathbb{E}[\gamma]\left(\sum_{m=1}^{M} \frac{\mathbb{E}\left[\lambda^{m-1}\right]}{\Gamma(\alpha m+1)} t^{\alpha m}\right)\left(\sum_{n=0}^{M} \frac{\mathbb{E}\left[\lambda^{n}\right]}{\Gamma(\alpha n+1)} t^{\alpha n}\right) \\
& +\mathbb{E}\left[\gamma^{2}\right] \sum_{m=1}^{M} \sum_{n=1}^{M} \frac{\mathbb{E}\left[\lambda^{m+n-2}\right]}{\Gamma(\alpha m+1) \Gamma(\alpha n+1)} t^{\alpha(m+n)} \\
& -(\mathbb{E}[\gamma])^{2}\left(\sum_{m=1}^{M} \frac{\mathbb{E}\left[\lambda^{m-1}\right]}{\Gamma(\alpha m+1)} t^{\alpha m}\right)\left(\sum_{n=1}^{M} \frac{\mathbb{E}\left[\lambda^{n-1}\right]}{\Gamma(\alpha n+1) t^{n}} t^{\alpha n}\right) .
\end{aligned}
$$

\section{Some illustrative examples}

This section is devoted to show two examples in order to illustrate all the theoretical results previously established. In particular, through the subsequent examples we want to highlight two key features of our study. Firstly, the method works successfully when $\lambda$ is a RV that belongs to the class $\mathbb{C}$ introduced in Definition 3. Specifically, in the first example (Example 2) we will consider that $\lambda$ is a bounded RV, thus it belongs to the class $\mathbb{C}$ (see Remark 6). Secondly, the technique can also be applied to obtain reliable approximations when $\lambda$ is an unbounded RV 
and it is approximated by an appropriate truncated (thus bounded) RV. This approach is very useful from a practical standpoint since explicit closed expressions for the statistical absolute moments of many RVs are not available. In such cases, checking condition (29) is either very difficult or simply impossible. This issue will be illustrated in the second example (Example 3 ). Additionally, in the Example 2 we will further check that the convergence of the mean and the variance (equivalently, the standard deviation) take place over the whole real line, i.e., for every $t \in \mathbb{R}$. Although this fact is already known from the theoretical results previously established, we think that the analysis is very instructive.

Example 2. Let us consider the random fractional IVP (18) where $\beta_{0}$ and $\gamma$ are 2-RVs such that

$$
\mathbb{E}\left[\beta_{0}\right]=\mathbb{E}[\gamma]=\frac{1}{2}, \quad \mathbb{V}\left[\beta_{0}\right]=\mathbb{V}[\gamma]=\frac{1}{2} .
$$

Observe that, for the sake of generality instead of fixing specific probability distributions for the RVs $\beta_{0}$ and $\gamma$, we have only specified values of their mean and variance. Hence, bounded and unbounded $R V$ s like a uniform $R V$ on the interval $\left[\frac{1-\sqrt{6}}{2}, \frac{1+\sqrt{6}}{2}\right]$; a gamma $R V$ of parameters $\left(r_{1} ; r_{2}\right)=(1 / 2 ; 1)$, and a gaussian $R V$ of parameters $\left(\mu ; \sigma^{2}\right)=(1 / 2 ; 1 / 2)$, are allowed to play the role of both $R V s$, for example. Furthermore, we will assume that $\lambda$ has a beta distribution of parameters $\left(b_{1} ; b_{2}\right)=(3 / 4 ; 1)$, i.e., $\lambda \sim B e(3 / 4 ; 1)$, hence $\lambda$ is a bounded $R V$ on the interval $[0,1]$ and, as a consequence, it belongs to the class $\mathbb{C}$ introduced in Definition 3 (see Remark 6). We will assume that all the input data $\beta_{0}, \gamma$ and $\lambda$ are statistically independent RVs. Therefore, hypotheses $\boldsymbol{H 1}-\boldsymbol{H} 2$ hold and it is then guaranteed that the approximation $Y_{M}(t)$, defined in (35) via a random generalized power series, will converge in the mean square sense to the exact solution $S P, Y(t)$. Accordingly, both the mean and the variance (or equivalently, the standard deviation) of the solution SP, $Y(t)$, to the random IVP (18) can be approximated using the expressions given in (38)-(39).

In Fig. 1, we have plotted the approximations of the mean and the standard deviation of the solution SP to the random IVP (18) with $\alpha=0.7$ using different orders of truncations $M \in$ $\{6,7,8,9,10,12,15,17,20\}$. For the sake of clarity in the graphical representation, we have shown the results over two different time intervals $[0,5]$ and $[0,10]$. From these plots, we observe that in order to get better approximations over larger intervals the order of truncation M must be higher.

It is known from our previous theoretical development (see Section 5) that these approximations of order $M$ for the mean and the standard deviation will converge all over the whole real line as $M \rightarrow+\infty$. Nevertheless, it is instructive to check this general result in the context of this example. With this aim, below we shall check this fact. Firstly, let us recall that the explicit value of higher moments of the beta distribution of parameters $\left(b_{1} ; b_{2}\right)$

$$
\mathbb{E}\left[\lambda^{m}\right]=\prod_{n=0}^{m-1} \frac{b_{1}+n}{b_{1}+b_{2}+n}, \quad \lambda \sim \operatorname{Be}\left(b_{1} ; b_{2}\right),
$$

satisfies the following first-order recurrence relationship

$$
\mathbb{E}\left[\lambda^{m+1}\right]=\frac{b_{1}+m}{b_{1}+b_{2}+m} \mathbb{E}\left[\lambda^{m}\right]
$$

Let us denote by

$$
e_{m}^{1}(t):=\frac{\mathbb{E}\left[\lambda^{m}\right]}{\Gamma(\alpha m+1)} t^{\alpha m}
$$


the general term of the first deterministic series that defines the approximation of order $M$ for the expectation of the solution SP (see (38)). Then, applying the ratio test for numerical series and using (42) and (34), one gets

$$
\begin{aligned}
\lim _{m \rightarrow+\infty} \frac{e_{m+1}^{1}(t)}{e_{m}^{1}(t)} & =\lim _{m \rightarrow+\infty} \frac{\mathbb{E}\left[\lambda^{m+1}\right]}{\mathbb{E}\left[\lambda^{m}\right]} \frac{\Gamma(\alpha m+1)}{\Gamma(\alpha(m+1)+1)} t^{\alpha} \\
& =\lim _{m \rightarrow+\infty}\left(\frac{b_{1}+m}{b_{1}+b_{2}+m}\right) \lim _{m \rightarrow+\infty}\left(\frac{1}{(\alpha(m+1))^{\alpha}} \sqrt{\frac{m}{m+1}}\right) t^{\alpha}=0,
\end{aligned}
$$

for every $t \in \mathbb{R}$ arbitrary but fixed. Following an analogous calculation, it can be seen the second sum in (38) converges over the whole real line as $M \rightarrow+\infty$. Observe that the above reasoning proves the convergence of the approximation for the mean given in (38) not only for the particular choice $\lambda \sim B e(3 / 4 ; 1)$ but for any values $b_{1}$ and $b_{2}$ of the parameters to the beta distribution.

In order to check the convergence of the approximation of the variance over the whole real line in the context of this example, we will use the representation given in (40). Therefore, we must justify the convergence, of the several series that appear in (40), for all $t \in \mathbb{R}$. However, essentially there are two different types of such series, namely, single and double series. We shall prove the convergence of the double series by applying Proposition 5. Let us define the general term

$$
a_{m n}=\frac{\mathbb{E}\left[\lambda^{m+n}\right]}{\Gamma(\alpha m+1) \Gamma(\alpha n+1)} t^{\alpha(m+n)}, \quad m \geq 0=m_{0}, n \geq 0=n_{0}, t \in \mathbb{R} \text { fixed } .
$$

Since $\lambda$ has a beta distribution, observe that

$$
\mathbb{E}\left[\lambda^{m+n}\right]=\int_{0}^{1} \lambda^{m+n} f_{\lambda}(\lambda) \mathrm{d} \lambda \leq \int_{0}^{1} f_{\lambda}(\lambda) \mathrm{d} \lambda=1,
$$

being $f_{\lambda}(\lambda)$ the PDF of $\lambda$. Then, for $t \in \mathbb{R}$ fixed one gets

$$
\begin{aligned}
\sum_{m=0}^{M} \sum_{n=0}^{N}\left|a_{m n}\right| & =\sum_{m=0}^{M} \sum_{n=0}^{N} \frac{\mathbb{E}\left[\lambda^{m+n}\right]}{\Gamma(\alpha m+1) \Gamma(\alpha n+1)}|t|^{\alpha(m+n)} \\
& \leq \sum_{m=0}^{M} \sum_{n=0}^{N} \frac{|t|^{\alpha m}}{\Gamma(\alpha m+1)} \frac{|t|^{\alpha n}}{\Gamma(\alpha n+1)} \\
& =\left(\sum_{m=0}^{M} \frac{|t|^{\alpha m}}{\Gamma(\alpha m+1)}\right)\left(\sum_{n=0}^{N} \frac{|t|^{\alpha n}}{\Gamma(\alpha n+1)}\right) \\
& \leq\left(\sum_{m \geq 0} \frac{|t|^{\alpha m}}{\Gamma(\alpha m+1)}\right)\left(\sum_{n \geq 0} \frac{|t|^{\alpha n}}{\Gamma(\alpha n+1)}\right) \\
& =\left(E_{\alpha, 1}\left(|t|^{\alpha}\right)\right)^{2}:=\alpha_{0}>0, \quad \forall M \geq m_{0}=0, \forall n \geq n_{0}=0,
\end{aligned}
$$

where in the last step we have used (28). Therefore, condition (i) of Proposition 5 holds. For the symmetry of the general term $a_{m n}$, it is sufficient to check condition (ii) of Proposition 5 for the rows, for instance. Let us take $n=\hat{n} \geq 0$ arbitrary but fixed, and let us consider the infinite series

$$
\sum_{m \geq 0} \hat{a}_{m}(t), \quad \hat{a}_{m}(t):=\frac{\mathbb{E}\left[\lambda^{m+\hat{n}}\right]}{\Gamma(\alpha m+1) \Gamma(\alpha \hat{n}+1)}|t|^{\alpha(m+\hat{n})} .
$$



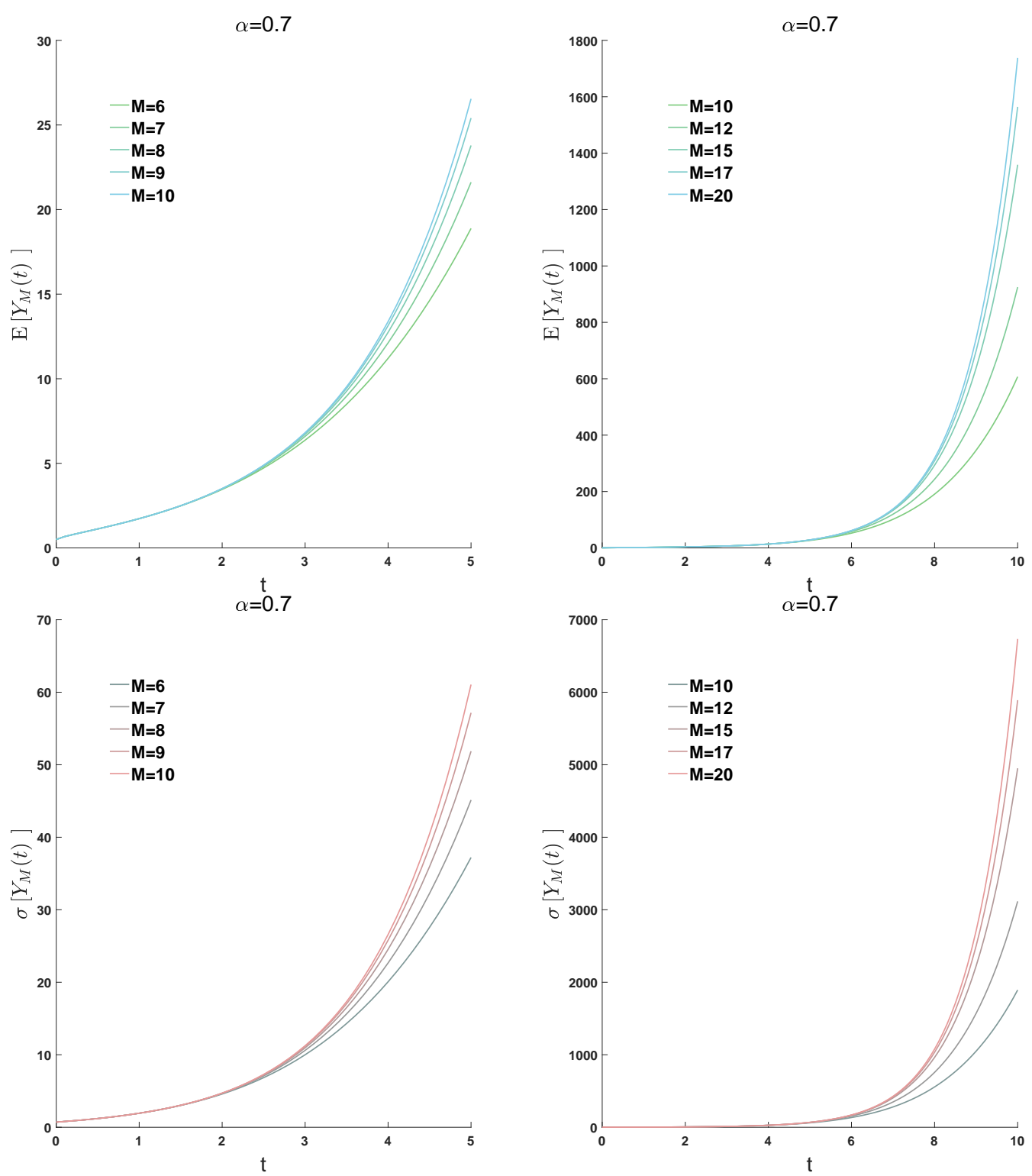

Figure 1: Approximations of the mean (top) and the standard deviation (bottom) of the solution SP to the random IVP (18) with $\alpha=0.7$ using different orders of truncations $M$ over the time intervals $[0,5]$ and $[0,10]$ in the context of Example 2. 
Since

$$
\begin{aligned}
\lim _{m \rightarrow+\infty} \frac{\hat{a}_{m+1}(t)}{\hat{a}_{m}(t)} & =\lim _{m \rightarrow+\infty} \frac{\mathbb{E}\left[\lambda^{m+\hat{n}+1}\right]}{\mathbb{E}\left[\lambda^{m+\hat{n}}\right]} \frac{\Gamma(\alpha m+1) \Gamma(\alpha \hat{n}+1)}{\Gamma(\alpha m+\alpha+1) \Gamma(\alpha \hat{n}+1)} \frac{|t|^{\alpha(m+\hat{n}+1)}}{|t|^{\alpha(m+\hat{n})}} \\
& =\left(\lim _{m \rightarrow+\infty} \frac{b_{1}+m+\hat{n}}{b_{1}+b_{2}+m+\hat{n}}\right)\left(\lim _{m \rightarrow+\infty} \frac{1}{(\alpha(m+1))^{\alpha}} \sqrt{\frac{m}{m+1}}\right)|t|^{\alpha}=0, \quad \forall t \in \mathbb{R},
\end{aligned}
$$

where we have used (34) and (42). The convergence of the second kind of infinite series can be checked directly taking advantage of the previous reasoning. Indeed, let us directly observe that

$$
\sum_{m \geq 0} \frac{\mathbb{E}\left[\lambda^{m}\right]}{\Gamma(\alpha m+1)} t^{\alpha m},
$$

and the convergence of this series follows using the same argument showed in (43)-(44).

In order to complete the probabilistic description of the solution SP to the fractional IVP (18), in Fig. 2 we have represented the correlation coefficient function of the approximation of order $M$

$$
\rho_{Y_{M}}(t, s)=\frac{\mathbb{C}_{Y_{M}, Y_{M}}(t, s)}{\sqrt{\mathbb{V}\left[Y_{M}(t)\right]} \times \sqrt{\mathbb{V}\left[Y_{M}(s)\right]}} .
$$

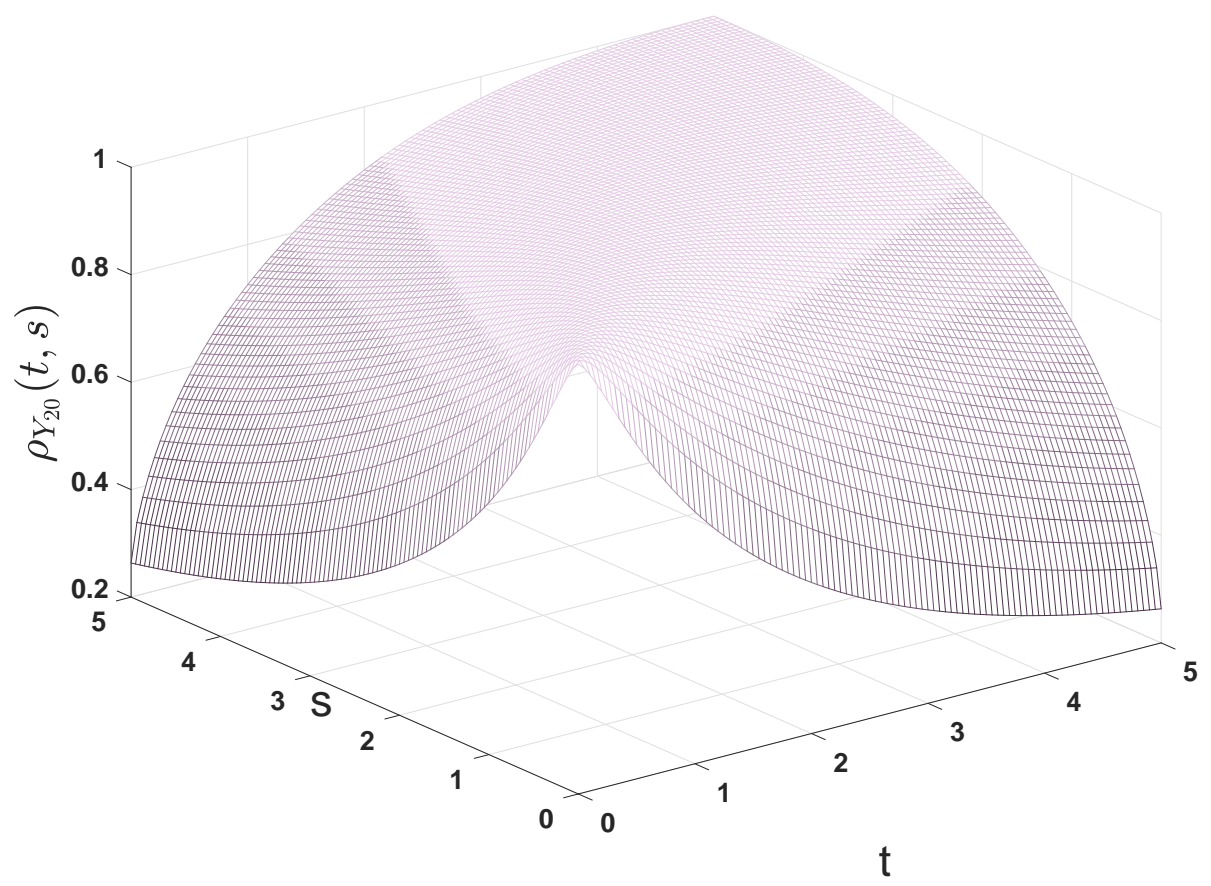

Figure 2: Correlation coefficient function $\rho_{Y_{M}}(t, s)$ of the approximation $Y_{M}(t)$ of order $M=20$ of the solution SP $Y(t)$ to the random $\operatorname{IVP}(18)$ with $\alpha=0.7$ over the time domain $(t, s) \in[0,5] \times[0,5]$ in the context of Example 2 . 
In Fig. 3, we have represented the approximations of the mean and standard deviation of the solution SP for different values of the differentiation parameters $\alpha=\{0.1,0.2, \ldots, 0.9,1\}$ taking as order of truncation $M=20$ over the time interval $[0,5]$. The plot of the mean provides a nice picture of the manner the solution SP varies as the fractional differentiation parameter changes from 0.1 to 1 . It is interesting to observe that the value of $\alpha=1$ corresponds to the classical first derivative. Thus, in that case the plot shows the mean of solution SP to the classical random IVP associated to (18), i.e.,

$$
\left\{\begin{array}{cl}
Y^{\prime}(t)-\lambda Y(t) & =\gamma, \quad t>0 \\
Y(0) & =\beta_{0}
\end{array}\right.
$$

We finish this example exhibiting a critical analysis about the computation of the order of truncation $M$ required so that, given an admissible error $\epsilon>0$, the finite numerical series approximation of the mean, given in (36), is uniformly bounded by $\epsilon$ in a bounded domain. Our next critical reflection can also be extended to the standard deviation. Let $b_{0}=\left|\mathbb{E}\left[\beta_{0}\right]\right|$ and $c=|\mathbb{E}[\gamma]|$ and assume that

$$
\exists q \in(0,1): H|t|^{\alpha}<q,
$$

being $H$ the positive constant associated to the $R V \lambda$, that is assumed to satisfy condition (29). Observe that applying (36), (29) and (45), one gets

$$
\begin{aligned}
\left|\mathbb{E}[Y(t)]-\mathbb{E}\left[Y_{M}(t)\right]\right| & =\left|\mathbb{E}\left[\beta_{0}\right] \sum_{m=M+1}^{\infty} \frac{\mathbb{E}\left[\lambda^{m}\right]}{\Gamma(\alpha m+1)} t^{\alpha m}+\mathbb{E}[\gamma] \sum_{m=M+1}^{\infty} \frac{\mathbb{E}\left[\lambda^{m-1}\right]}{\Gamma(\alpha m+1)} t^{\alpha m}\right| \\
& \leq \sum_{m=M+1}^{\infty} \frac{b_{0} \mathbb{E}\left[|\lambda|^{m}\right]+c \mathbb{E}\left[|\lambda|^{m-1}\right]}{\Gamma(\alpha m+1)}|t|^{\alpha m} \\
& \leq \sum_{m=M+1}^{\infty} \frac{b_{0} L H^{m}+c L H^{m-1}}{\Gamma(\alpha m+1)}|t|^{\alpha m} \\
& =\sum_{m=M+1}^{\infty} \frac{\left(b_{0}+\frac{c}{H}\right) L H^{m}}{\Gamma(\alpha m+1)}|t|^{\alpha m} \\
& =\left(b_{0}+\frac{c}{H}\right) L \sum_{m=M+1}^{\infty} \frac{\left(H|t|^{\alpha}\right)^{m}}{\Gamma(\alpha m+1)} \\
& =\left(b_{0}+\frac{c}{H}\right) L \sum_{m=M+1}^{\infty} \frac{q^{m}}{\Gamma(\alpha m+1)} \\
& =\left(b_{0}+\frac{c}{H}\right) L \sum_{m=M+1}^{\infty} q^{m} \\
& =\left(b_{0}+\frac{c}{H}\right) L \frac{q^{M+1}}{1-q} .
\end{aligned}
$$

Therefore, given an admissible error $\epsilon>0$, if we take the order of truncation so that

$$
M \geq\left[\frac{\ln \left(\frac{\epsilon(1-q) H}{\left(b_{0} H+c\right) L}\right)}{\ln (q)}-1\right]+1,
$$



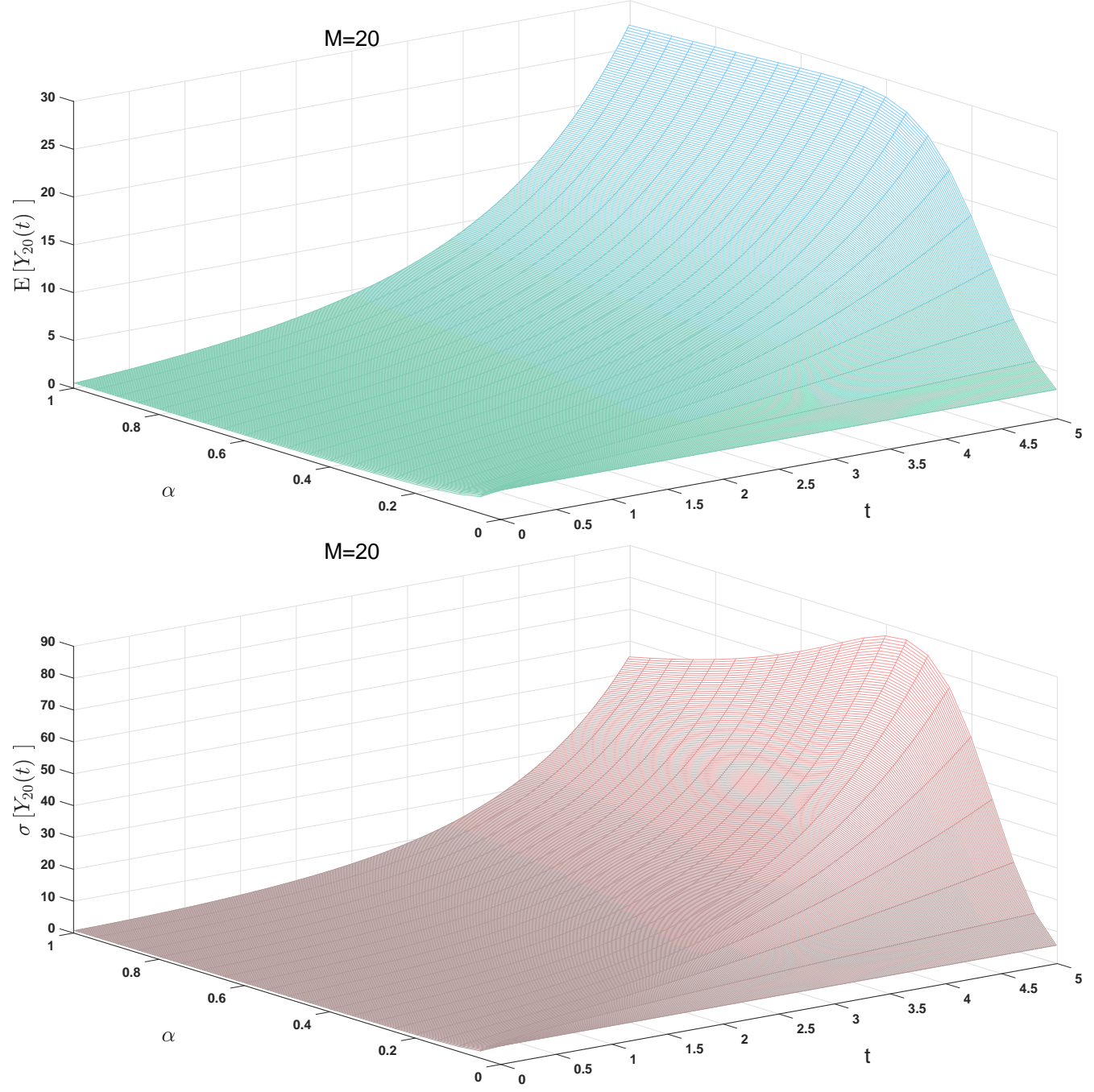

Figure 3: Approximations of the mean (top) and the standard deviation (bottom) of the solution SP to the random IVP (18) varying the fractional differentiation parameter $\alpha=\{0.1,0.2, \ldots, 0.9,1\}$ taking as order of truncation $M=20$ over the time interval $[0,5]$ in the context of Example 2. 
where [.] stands for the ceiling function, then it is guaranteed that

$$
\left|\mathbb{E}[Y(t)]-\mathbb{E}\left[Y_{M}(t)\right]\right|<\epsilon, \quad \forall|t|<\left(\frac{q}{H}\right)^{1 / \alpha} .
$$

In Table 1, we show the theoretical values for the order of truncation M computed by (47) taking the same numerical data used to construct the approximations of the mean that we have plotted in Figure 1, i.e., $\alpha=0.7, b_{0}=c=1 / 2$. Besides, observe that the values $L=H=1$ and $H$ satisfy condition (29). Indeed, they can be easily deduced since for the beta $R V \lambda, \mathbb{E}\left[|\lambda|^{m}\right] \leq 1$ for all $m \geq 0$. The figures collected in Table 1 have been determined over the domain (45) with $q=0.9$, i.e.,

$$
0<|t|<\left(\frac{0.9}{1}\right)^{1 / 0.7}=0.8602648
$$

for different admissible errors $\epsilon>0$. In Table 1 we also compare the theoretical values of $M$ with those, denoted by $\hat{M}$, obtained from directed computations. Specifically, $\hat{M}$ has been computed as the first value so that

$$
\left|\mathbb{E}\left[Y_{\hat{M}}(t)\right]-\mathbb{E}\left[Y_{\hat{M}-1}(t)\right]\right|<\epsilon, \quad \forall 0<|t|<0.8602648,
$$

for a given value of $\epsilon>0$. We can observe that the values of $M$ are very conservative estimates. The determination of the order of truncation $M$ given in (47) has been based on majorizing the error by a geometric series (see (46)). This restricts the analysis to the domain (45) (or equivalently to (48)), which is contained within the unit interval $(0,1)$. Using appropriate bounds for the remainder of Mittag-Lefler type-series (see (28)), $\sum_{m \geq M+1} z^{m} / \Gamma(\alpha m+v)$, the above analysis can be carried out for the complementary domain of (45). Such appropriate bounds can be found from the results shown in [31, ch.4]. Although, interesting from a theoretical standpoint, these results have a limited value in practice, as it has already been pointed out.

\begin{tabular}{|c|c|c|c|c|c|}
\hline & $\epsilon=10^{-3}$ & $\epsilon=10^{-4}$ & $\epsilon=10^{-5}$ & $\epsilon=10^{-6}$ & $\epsilon=10^{-7}$ \\
\hline$M$ & 87 & 109 & 131 & 152 & 174 \\
\hline$\hat{M}$ & 8 & 10 & 11 & 12 & 14 \\
\hline
\end{tabular}

Table 1: Theoretical values for the order of truncation $M$ using different values of the admissible error $\epsilon>$ in the context of Example 2. These theoretical values are compared with those, denoted by $\hat{M}$, obtained directly from our numerical computations.

Example 3. In Remark 6 it has been pointed out that the truncation method is a useful technique to approximate unbounded RVs [35, ch.V]. In practice, this approach is preferable that checking condition (29) for particular probability distributions assigned to the RV $\lambda$. Indeed, this latter idea could be even unaffordable since there are RVs, such as binomial RVs, for which a closed expression for their statistical moments are not available. Motivated by fact, this example has been devised to illustrate the capability of the proposed method to compute reliable approximations of the mean and the standard deviations of the solution SP to the random IVP (18), in the case that the $R V \lambda$ is unbounded but it is approximated by means of appropriate truncation. With this aim, let us assume that $\lambda$ is an exponential $R V$ of mean $1 / \lambda_{0}$, i.e., $\lambda \sim \operatorname{Exp}\left(\lambda_{0}\right)$ and let us consider its probabilistic approximation using the truncation method. We thus approximate the 
exponential $R V \lambda$ by means of another exponential $R V$, say $\hat{\lambda} \sim \operatorname{Exp}\left(\hat{\lambda}_{0}\right)$, defined on the finite interval $[0, a], a>0$, so that both $R V s, \lambda$ and $\hat{\lambda}$, have the same mean

$$
\frac{1}{\lambda_{0}}=\mathbb{E}[\lambda]=\mathbb{E}[\hat{\lambda}] .
$$

The PDF of $R V \hat{\lambda}$ is

$$
f_{\hat{\lambda}}(\hat{\lambda})=\frac{\hat{\lambda}_{0} \exp \left(-\hat{\lambda}_{0} \hat{\lambda}\right)}{\int_{0}^{a} \hat{\lambda}_{0} \exp \left(-\hat{\lambda}_{0} \hat{\lambda}\right) \mathrm{d} \hat{\lambda}}, \quad 0 \leq \hat{\lambda} \leq a .
$$

Now, we determine the value of the parameter $\hat{\lambda}_{0}$ satisfying condition (49)

$$
\frac{1}{\lambda_{0}}=\frac{\int_{0}^{a} \hat{\lambda} \hat{\lambda}_{0} \exp \left(-\hat{\lambda}_{0} \hat{\lambda}\right) \mathrm{d} \hat{\lambda}}{\int_{0}^{a} \hat{\lambda}_{0} \exp \left(-\hat{\lambda}_{0} \hat{\lambda}\right) \mathrm{d} \hat{\lambda}} .
$$

In our numerical experiments we have taken $a=10$ and $\lambda_{0}=2$. Thus, according to (51) $\lambda_{0}$ is the root of the following nonlinear equation

$$
1-\exp \left(-10 \hat{\lambda}_{0}\right)=\left(1-\exp \left(-10 \hat{\lambda}_{0}\right)\left(1+10 \hat{\lambda}_{0}\right)\right) \hat{\lambda}_{0} .
$$

${ }_{51}$ Using a numerical iterative method it can be checked that $\hat{\lambda}_{0}=1.9999999175537901$ is the solution of (52). In order to demonstrate the reliability of the approximations obtained for the mean and the standard deviation using the approach previously described, we have computed the relative error for the mean, $R E(M e a n)$, and for the standard deviation, $R E(S D)$. These relative errors have been calculated using the following expressions

$$
R E(\text { Mean })=R E(\text { Mean })(t ; M)=\frac{\left|\mathbb{E}\left[\hat{Y}_{M}(t)\right]-\mathbb{E}[Y(t)]\right|}{\mathbb{E}[Y(t)]},
$$

$$
R E(S D)=R E(S D)(t ; M)=\frac{\left|\sqrt{\mathbb{V}\left[\hat{Y}_{M}(t)\right]}-\sqrt{\mathbb{V}[Y(t)]}\right|}{\sqrt{\mathbb{V}[Y(t)]}}
$$

where $\mathbb{E}\left[\hat{Y}_{M}(t)\right]$ and $\mathbb{V}\left[\hat{Y}_{M}(t)\right]$ are the approximation of the mean and the variance, respectively, of the solution s.p. $Y(t)$ at the time point $t$ using the expression (38) and (40), respectively, with $\alpha=0.7, \mathbb{E}\left[\beta_{0}\right]=\mathbb{E}[\gamma]=0.5$, as in the Example 1 (see (41)), and using the bounded $R V$, $\hat{\lambda} \sim \operatorname{Exp}\left(\hat{\lambda}_{0}=1.9999999175537901\right.$ defined on the finite interval $[0,6]$. Therefore, the higher moments of $\hat{\lambda}$, that appear in (38), have been computed by

$$
\mathbb{E}\left[\hat{\lambda}^{m}\right]=\int_{0}^{6} \hat{\lambda}^{m} f_{\hat{\lambda}}(\hat{\lambda}) \mathrm{d} \hat{\lambda},
$$

being $f_{\hat{\lambda}}(\hat{\lambda})$ defined in (50). While the exact mean and variance of $Y(t)$ in (53) and (54), denoted by $\mathbb{E}[Y(t)]$ and $\mathbb{V}[Y(t)]$, respectively, have been computed using (38) and (40) by taking $\lambda \sim$ $\operatorname{Exp}(\hat{\lambda}=2)$ and $M=20$, for which the numerical stabilization of approximations has been checked to be exact up to the nine first decimals digits.

In Tables 2 and 3 we show the numerical results for both relative errors. From the figures collected in these tables we can see that the approximations for the mean and standard deviations obtained using the proposed truncated method are very accurate. As it is expected, these approximations improve as $M$ increases for $t$ fixed, while the accuracy decreases as $t$ departs from the origin for $M$ fixed. 


\begin{tabular}{|c|c|c|c|c|c|}
\hline RE(Mean)(t;M) & $t=0.1$ & $t=0.3$ & $t=0.5$ & $t=0.7$ & $t=0.9$ \\
\hline $\mathrm{M}=5$ & $2.7235 \cdot 10^{-5}$ & $2.9732 \cdot 10^{-3}$ & $3.6638 \cdot 10^{-2}$ & $3.1118 \cdot 10^{-1}$ & $8.4798 \cdot 10^{-1}$ \\
\hline $\mathrm{M}=7$ & $1.3773 \cdot 10^{-6}$ & $7.4927 \cdot 10^{-4}$ & $2.0926 \cdot 10^{-2}$ & $2.7211 \cdot 10^{-1}$ & $8.2723 \cdot 10^{-1}$ \\
\hline $\mathrm{M}=10$ & $1.6241 \cdot 10^{-8}$ & $1.2648 \cdot 10^{-4}$ & $1.077 \cdot 10^{-2}$ & $2.2707 \cdot 10^{-1}$ & $7.8983 \cdot 10^{-1}$ \\
\hline $\mathrm{M}=12$ & $3.6711 \cdot 10^{-9}$ & $4.4072 \cdot 10^{-5}$ & $7.2343 \cdot 10^{-3}$ & $1.9819 \cdot 10^{-1}$ & $7.5255 \cdot 10^{-1}$ \\
\hline $\mathrm{M}=15$ & $5.1902 \cdot 10^{-9}$ & $9.9181 \cdot 10^{-6}$ & $3.7974 \cdot 10^{-3}$ & $1.4757 \cdot 10^{-1}$ & $6.4953 \cdot 10^{-1}$ \\
\hline
\end{tabular}

Table 2: Relative error for the mean $\operatorname{RE}(\operatorname{Mean})(t ; M)$ computed by (53) for different values of $t$ and $M$ in the context of Example 3.

\begin{tabular}{|c|c|c|c|c|c|}
\hline $\mathrm{RE}(\mathrm{SD})(\mathrm{t} ; \mathrm{M})$ & $t=0.1$ & $t=0.3$ & $t=0.5$ & $t=0.7$ & $t=0.9$ \\
\hline $\mathrm{M}=5$ & $8.2462 \cdot 10^{-5}$ & $4.3389 \cdot 10^{-1}$ & $9.9813 \cdot 10^{-1}$ & $9.9960 \cdot 10^{-1}$ & $9.9999 \cdot 10^{-1}$ \\
\hline $\mathrm{M}=7$ & $6.4702 \cdot 10^{-6}$ & $3.9683 \cdot 10^{-1}$ & $9.9741 \cdot 10^{-1}$ & $9.9991 \cdot 10^{-1}$ & $9.9999 \cdot 10^{-1}$ \\
\hline $\mathrm{M}=10$ & $1.8467 \cdot 10^{-7}$ & $6.3619 \cdot 10^{-1}$ & $9.9424 \cdot 10^{-1}$ & $9.9953 \cdot 10^{-1}$ & $9.9992 \cdot 10^{-1}$ \\
\hline $\mathrm{M}=12$ & $1.4535 \cdot 10^{-8}$ & $3.8055 \cdot 10^{-1}$ & $9.8742 \cdot 10^{-1}$ & $9.9826 \cdot 10^{-1}$ & $9.9959 \cdot 10^{-1}$ \\
\hline $\mathrm{M}=15$ & $6.0470 \cdot 10^{-9}$ & $3.3668 \cdot 10^{-1}$ & $9.4612 \cdot 10^{-1}$ & $9.8411 \cdot 10^{-1}$ & $9.9352 \cdot 10^{-1}$ \\
\hline
\end{tabular}

Table 3: Relative error for the standard deviation $\operatorname{RE}(\mathrm{SD})(t ; M)$ computed by (54) for different values of $t$ and $M$ in the context of Example 3.

\section{Conclusions}

In the first part of this paper we have extended to the random framework the deterministic Riemann-Liouville integral and Caputo derivative. This extension has been done in the Banach space $\left(\mathrm{L}^{2}(\Omega),\|\cdot\|_{2}\right)$ of the random variables and stochastic process of second-order, i.e., having finite variance. This condition is often met for the majority of physical phenomena. An important advantage of the aforementioned extension is that it remains valid for other Banach spaces $\left(\mathrm{L}^{p}(\Omega),\|\cdot\|_{p}\right), p \geq 2$. Furthermore, an additional benefit of our approach is that our results have been established using a strong stochastic convergence, namely the mean square convergence. Therefore, our results are also valid when using another type of weaker stochastic convergences, such that the convergence in probability and in distribution, which are used in many contexts. In the second part of the paper, we have taken advantage of the results established in the first part together with a mean square chain rule for differentiating second-order stochastic processes, to construct a solution stochastic process of the general random linear fractional differential equation assuming mild conditions of the random inputs (initial condition, forcing term and diffusion coefficient). Furthermore, we have given general explicit expressions for constructing reliable approximations of the mean, variance and covariance of the solution stochastic process. Finally, we have illustrated our main theoretical findings and the potentiality of our approach through two examples. We expect the results and ideas provided in this contribution can be useful in forthcoming extension of random fractional differential equations using the mean square random calculus.

\section{Acknowledgements}

This work has been partially supported by the Ministerio de Economía y Competitividad grant MTM2013-41765-P. The co-author Prof. L. Villafuerte acknowledges the support by Mex- 
ican Conacyt.

\section{Conflict of Interest Statement}

The authors declare that there is no conflict of interests regarding the publication of this article.

\section{References}

[1] R. C. Smith, Uncertainty Quantification. Theory, Implementation and Applications, Computational Science and Engineering, SIAM, Philadelphia, 2014.

[2] L. Arnold, Stochastic Differential Equations: Theory and Applications, Krieger Publishing Company, New York, 1992.

[3] B. Øksendal, Stochastic Differential Equations: An Introduction with Applications, Springer-Verlag, BerlinHeidelberg, 2003.

[4] P. Kloeden, E. Platen, Numerical Solution of Stochastic Differential Equations, Applications of Mathematics, Springer, Berlin, 1992.

[5] T. Neckel, F. Rupp, Random Differential Equations in Scientific Computing, Versita, London, 2013.

[6] R. G. Ghanem, P. D. Spanos, Stochastic Finite Elements: A Spectral Approach, Courier Dover Publ., New York, 2003.

[7] D. Xiu, Numerical Methods for Stochastic Computations. A Spectral Method Approach, Princeton University Press, New Jersey, 2010

[8] T. T. Soong, Random Differential Equations in Science and Engineering, Academic Press, New York, 1973.

[9] D. Xiu, G. E. Karniadakis, Wiener-Askey polynomial chaos for stochastic differential equations, SIAM Journal on Scientific Computing 24 (2) (2002) 619-644.

[10] F. A. Dorini, M. S. Cecconello, M. B. Dorini, On the logistic equation subject to uncertainties in the environmental carrying capacity and initial population density, Communications in Nonlinear Science and Numerical Simulation 33 (2016) 160-173. doi:10.1016/j.cnsns.2015.09.009.

[11] M. M. R. Williams, Polynomial chaos functions and stochastic differential equations, Annals of Nuclear Energy 33 (9) (2006) 774-785.

[12] A. Hussein, M. M. Selim, Solution of the stochastic radiative transfer equation with Rayleigh scattering using RVT technique, Applied Mathematics and Computation 218 (13) (2012) 7193-7203. doi:10.1016/j.amc.2011.12.088.

[13] A. K. Golmankhaneh, N. A. Porghoveh, D. Baleanu, Mean square solutions of second-order random differential equations by using homotopy analysis method, Romanian Reports in Physics 65 (2013) 350-362.

[14] K. Nouri, H. Ranjbar, Mean square convergence of the vnumerical solution of random differential equations, Mediterranean Journal of Mathematics 12 (2015) 1123-1140. doi:10.1007/s00009-014-0452-8.

[15] M. Khodabin, M. Rostami, Mean square numerical solution of stochastic differential equations by fourth order Runge-Kutta method and its application in the electric circuits with noise, Mediterranean Journal of Mathematics 62 (2015) 1-19. doi:10.1186/s13662-015-0398-6.

[16] M. Khodabin, K. Maleknejad, M. Rostami, M. Nouri, Numerical solution of stochastic differential equations by second order Runge-Kutta methods, Mathematical and Computer Modelling 53 (2011) 1910-1920. doi:10.1016/j.mcm.2011.01.018.

[17] A. A. Kilbas, H. M. Srivastava, J. J. Trujillo, Theory and Applications of Fractional Differential Equations, Elsevier Science, The Netherlands, 2006.

[18] Y. Khan, M. Fardi, K. Sayevand, M. Ghasemi, Solution of nonlinear fractional differential equations using an efficient approach, Neural Computing and Applications 24 (2014) 187-192. doi:10.1007/s00521-012-1208-7.

[19] A. K. Golmankhaneh, R. Arefi, D. Baleanu, Synchronization in a nonidentical fractional order of a proposed modified system, Journal of Vibration and Control 21 (2015) 1154-1161.

[20] M. Hahn, S. Umarov, Fractional Fokker-Planck-Kolmogorov type equations and their associated stochastic differential equations, Fractional Calculus and Applied Analysis 14 (1) (2011) 56-79. doi:10.2478/s13540-011-0005-9.

[21] T. Atanacković, M. Nedeljkov, S. Pilipović, D. Rajter-Ćiri, Dynamics of a fractional derivative type of a viscoelastic rod with random excitation, Fractional Calculus and Applied Analysis 18 (5) (2015) 1232-1251. doi:10.1515/fca2015-0071.

[22] V. Lupulescu, D. O’Reagan, G. u. Rahman, Existence results for random fractional differential equations, Opuscula Mathematica 34 (4) (2014) 813-825. doi:10.7494/OpMath.2014.34.4.813. 
[23] V. Lupulescu, K. N. Ntouyas, Random fractional differential equations, International Electronic Journal of Pure and Applied Mathematics 4 (2) (2012) 119-136.

[24] Y. Khan, Q. Wu, N. Faraz, A. Yildirim, M. Madani, New fractional analytical approach via a modified RiemannLiouville derivative, Applied Mathematics Letters 25 (2012) 1729-1733. doi:10.1016/j.aml.2012.02.001.

[25] G. González-Parra, B. M. Chen-Charpentier, A. J. Arenas, Polynomial chaos for random fractional order differential equations, Applied Mathematics and Computation 226 (2014) 123-130. doi:10.1016/j.amc.2013.10.051.

[26] M. Loève, Probability Theory II, Vol. 46 of Graduate Texts in Mathematics, Springer-Verlag, New York, 1978.

[27] L. Villafuerte, C. A. Braumann, J. C. Cortés, L. Jódar, Random differential operational calculus: Theory and applications, Computers and Mathematics with Applications 59 (1) (2010) 115-125. doi:10.1016/j.camwa.2009.08.061.

[28] G. R. Grimmett, D. R. Stirzaker, Probability and Random Processes, Clarendon Press, New York, 2000

[29] J. C. Cortés, L. Villafuerte, C. Burgos, A mean square chain rule and its applications in solving the random Chebyschev differential equation, arXiv https://arxiv.org/abs/1612.08639.

[30] J. C. Cortés, P. Sevilla-Peris, L. Jódar, Analytic-numerical approximating processes of diffusion equation with data uncertainty, Computers and Mathematics with Applications 49 (7-8) (2005) 1255-1266. doi:10.1016/j.camwa.2004.05.015.

[31] R. Gorenflo, A. A. Kilbas, F. Mainardi, S. V. Rogosin, Mittag-Leffler Functions, Related Topics and Applications, Springer-Verlag, Berlin Heidelberg, 2014. doi:10.1007/978-3-662-43930-2.

[32] M. Mursaleen, S. A. Mohiuddine, Convergence Methods for Double Sequences and Applications, Springer, India, 2014. doi:10.1007/978-81-322-1611-7.

[33] J. C. Cortés, L. Jódar, J. Camacho, L. Villafuerte, Random Airy type differential equations: Mean square exact and numerical solutions, Computers and Mathematics with Applications 60 (5) (2010) 1237-1244. doi:10.1016/j.camwa.2010.05.046.

[34] G. Calbo, J. C. Cortés, L. Jódar, L. Villafuerte, Solving the random Legendre differential equation: Mean square power series solution and its statistical functions, Computers and Mathematics with Applications 61 (9) (2011) 2782-2792. doi:10.1016/j.camwa.2011.03.045.

[35] M. Loève, Probability Theory I, Vol. 45 of Graduate Texts in Mathematics, Springer-Verlag, New York, 1977. 